\title{
MXene based new class of silicone oil nanofluids for the performance improvement of Concentrated Photovoltaic Thermal Collector
}

\author{
Navid Aslfattahi ${ }^{1, *}$, L Samylingam ${ }^{2}$, AS Abdelrazik ${ }^{3}$, A. Arifutzzaman ${ }^{2}$, \\ R. Saidur ${ }^{2,4, *}$ \\ ${ }^{1}$ Department of Mechanical Engineering, Faculty of Engineering, University of Malaya, 50603, Kuala Lumpur \\ ${ }^{2}$ Research Center for Nano-Materials and Energy Technology (RCNMET), School of Science and Technology, \\ Sunway University, Bandar Sunway, Petaling Jaya, 47500, Selangor Darul Ehsan, Malaysia \\ ${ }^{3}$ Mechanical Engineering Department, King Fahd University of Petroleum \& Minerals, Dhahran, Saudi Arabia \\ ${ }^{4}$ Department of Engineering, Lancaster University, Lancaster, LAl 4YW, UK
}

Corresponding authors: saidur@ sunway.edu.my, navid.fth87@yahoo.com

\section{Abstract}

In this research work, MXene with a chemical formula of $\mathrm{Ti}_{3} \mathrm{C}_{2}$ is used for the first time with silicone oil to improve thermo-physical properties of MXene based silicone oil. This paper focuses on preparation, characterization, thermal properties, thermal stability and performance investigation of new class of silicone oil nanofluids induced with MXene in three different concentrations for a Concentrated Solar Photovoltaic Thermal (CPVT) collector.

The thermal conductivity of the silicone oil-based MXene nanofluids is measured using a Transient Hot Bridge (THB) 500. Viscosity is measured using a Rheometer at various temperatures including 25, 50, 75, 100, and $125^{\circ} \mathrm{C}$. Perkin Elmer Lambda 750 is used to measure optical absorbance. The highest thermal conductivity enhancement is found to be $64 \%$ for $0.1 \mathrm{wt} \%$ concentration of silicone oil-MXene nanofluid compared to pure silicone oil at $150{ }^{\circ} \mathrm{C}$. The viscosity of MXene with silicone oil nanofluids is found to be independent of addition of MXene nanoparticles in the silicone oil base fluid. Viscosity is reduced by $37 \%$ when temperature is raised from $25^{\circ} \mathrm{C}$ to 50 ${ }^{\circ} \mathrm{C}$ for different concentrations of MXene with silicone oil . Silicone oil-based MXene nanofluid with 0.1 wt. $\%$ concentration is thermally stable up to $\sim 380{ }^{\circ} \mathrm{C}$. Introducing more MXene nanoparticles into silicone oil improves electrical efficiency of PV module due to better cooling of MXene based nanofluids. Higher solar concentration is resulted in higher average temperature of 
the PV module. This consequently raises thermal energy gain which is useful for different applications.

Keywords: MXene; Nanofluids; CPVT; thermo-physical properties

\section{Introduction}

When nano-sized particles are suspended into traditional heat transfer fluids, the term is called nanofluids [1]. Nanofluids have superior thermal properties due to the dispersion of nanoparticles $[2,3]$. Murshed, Leong [4] reported that there are data on thermal properties, thermal conductivity, and enhancement of nanofluids in the open literatures. Murshed, Leong [4] also stated that nanofluids enhanced thermal properties better than the base fluid. The stability of the dispersed nanoparticle is an important criterion to determine the thermal properties of a nanofluid [5]. Nevertheless, the percentage of nanoparticles into a base fluid in volume percentage will determine the stability and suspension of the nanofluid and the improvement of thermal properties $[6,7]$.

Many studies on the enhanced thermal properties of nanofluids were conducted in the past decade. Metals such as metal oxides, metal carbide, metal nitride, and carbon materials were categorized as nanoparticles [8-10]. To date, numerous research were carried out on $\mathrm{Cu}, \mathrm{Ag}, \mathrm{Ni}$, $\mathrm{Au}$ (metals), $\mathrm{Al}_{2} \mathrm{O}_{3}, \mathrm{CuO}, \mathrm{MgO}, \mathrm{ZnO}, \mathrm{SiO}_{2}, \mathrm{Fe}_{2} \mathrm{O}_{3}, \mathrm{TiO}_{2}$ (metal oxides), $\mathrm{SiC}$ (metal carbide), AlN (metal nitride), CNTs, MWCNTs, diamond and graphene (carbon materials) [8-10]. Among all the nanoparticles, the graphene is a 2-dimensional material that showed the highest thermal properties due to the largest surface area compared to other nano-materials.

Domestic and industrial sectors use concentrated photovoltaic thermal (CPVT) collectors and systems widely. High thermal and electrical outputs provided by CPVT collectors as the incoming sunlight and sun irradiation is maximized on the cell surface via energy-efficient concentrators $[11,12]$. When concentrated sunlight fall into the cell surface, high heat flux occurs and raise the heat transfer fluid's temperature in the system rapidly $[11,13]$. Conventional heat transfer fluids used in solar thermal collectors are limited by poor heat transfer properties [13]. Therefore, to improve the solar energy conversion efficiencies, researches have been replacing conventional heat transfer fluids with nanofluids which enhanced heat transfer properties $[14,15]$. According to Tyagi, Phelan [17], the efficiency of solar system enhanced by $10 \%$ by adding $\mathrm{Al}_{2} \mathrm{O}_{3}$ to the heat 
transfer fluids. Saidur, Meng [18] found out that the collector performance and solar absorption rate increased when the volume fraction of nanoparticle increased up to $1 \%$ in the fluid. Taylor, Phelan [19] found that the thermal performance of the solar efficiency increased by $10 \%$ with the addition of graphite nano-particles in the heat transfer fluids. Otanicar, Phelan [20] conducted experiment on the performance of the direct absorption solar collector (DASC) using various type of nanofluids (graphite, CNT and silver nanoparticles). The authors found out that the efficiency of DASC improved by $5 \%$ due to promising properties of nanofluids.

Using nanofluids from the front side of the solar systems has gained great interest among the many researchers to evaluate the influence of the optical properties of the nanofluid to show the effectiveness of using nanofluids on the enhancement of the efficiency of the Direct Absorption Solar Collectors (DASC). However, few studies investigated the application of the optical filtration, using nanofluids, in the hybrid PV/T and CPV/T systems. Han et al. [21] suspended silver (Ag) nanoparticles in a hybrid $\mathrm{CoSO}_{4}-\mathrm{PG}$ base fluid and the mixture was tested on the front side of the PV panel in a hybrid PV/T system. The authors observed that the filters showed high transmittance in the useful ranges of the cell material and higher absorbance outside the solar radiation ranges. Abdelrazik et al. [22] ] investigated experimental and numerical study on the influence of the optical filtration using water/Ag nanofluid on the performance of hybrid PV/T systems. Defining an equivalent electrical efficiency, they reported higher values for the PV/T system with optical filtration compared to the standalone PV system (24.5\% compared to $10.5 \%$ ) at low nanoparticles concentration $(0.0005$ wt.\%). However, the hybrid system was electricallyinefficient at high nanoparticles concentrations (>0.5 wt.\%). In another study, Abdelrazik et al. [23] mentioned that the strength of the optical filtration in the PV/T systems came from the filtration, from one side, and the cooling, from another side, that it provides at the same time.

Crisostomo et al. [24] prepared an optical filtration nanofluid using the core-shell $\mathrm{Ag}-\mathrm{SiO}_{2}$ dispersed in water base fluid. Under the same illumination conditions, the results showed $12 \%$ improvement in the weighted energy output from a PV/T system, using the water/Ag- $\mathrm{SiO}_{2}$ for optical filtration, in comparison to a standalone PV system. An et al. [25] ] carried out an outdoor experimental testing for the performance a hybrid CPV/T system accompanied with optical filtration, using a solution from the $\mathrm{Cu}_{9} \mathrm{~S}_{5}$ nanoparticles. They reported that the overall efficiency 
of their system, with optical filtration, was $17.9 \%$ better than the standalone system without filtration.

Emerging nano-materials, MXene was invented by Drexel University in 2011 by Naguib et al. [26]. Since then an extensive number of experimental and theoretical studies were carried out on this material due to its superior properties compared to some other materials. This material found to have better thermal, electrical, optical and other properties along with their versatile applications as reported in literature. MXenes family materials inclusive a general formula of $M_{n+1} X_{n} T_{x}(n=1$ 3), where $\mathrm{M}$ indicates an early transition metal (Ti, Sc, V, Cr, Ta, Nb, Zr, Mo, Hf), X stands for C and/or $\mathrm{N}$ atoms and $\mathrm{T}_{\mathrm{x}}$ represents the surface terminations $\left(\_\mathrm{O},{ }_{-} \mathrm{OH}\right.$, and _F) which are attached to the surface of the MXene nanomaterial. In MXenes family materials, $n+1$ layers of M cover $n$ layers of $\mathrm{X}$ in an $[\mathrm{MX}]_{\mathrm{n}} \mathrm{M}$ arrangements. The first $\mathrm{MXene}$ family material $\left(\mathrm{Ti}_{3} \mathrm{C}_{2}\right)$ was synthesized in 2011, using hydrofluoric acid (HF) etching process [26]. More MXene nanomaterials have been synthesized using different wet-chemistry etching methods and more MXenes family materials are expected to discover. In addition, it has a large surface area, hydrophilicity, adsorption ability, and high surface reactivity, which are useful for various energy and other applications [27].

MXenes are derived from transition metals such as, carbides, nitrides, and carbonitrides [26]. Till to date, even though there are approximately 70 MAX phases discovered, only a few MXenes have been established using etching method. The established MXenes are $\mathrm{Ti}_{3} \mathrm{C}_{2}, \mathrm{Ti}_{2} \mathrm{C},\left(\mathrm{Ti}_{0.5}\right.$, $\left.\mathrm{Nb}_{0.5}\right)_{2} \mathrm{C},\left(\mathrm{V}_{0.5}, \mathrm{Cr}_{0.5}\right)_{3} \mathrm{C}_{2}, \mathrm{Ti}_{3} \mathrm{CN}, \mathrm{Ta}_{4} \mathrm{C}_{3}$ [28], $\mathrm{Nb}_{2} \mathrm{C}, \mathrm{V}_{2} \mathrm{C}$ [29], and $\mathrm{Nb}_{4} \mathrm{C}_{3}$ [30]. In order to produce emerging nanoparticles, the layers of SP elements from 3-dimensional materials (MAX phases) are etched. Selective etching method is used to remove "A" layers from MAX phases to produce MXene multilayer flakes. Details of this material such as synthesis, properties and applications are provided comprehensively in one of the review article [31]. MXenes are unique and better than other nanoparticles in terms of energy storage properties, high capacitance, good electrical conductivity, and high mechanical properties. These excellent properties made MXenes as good candidates for many applications such as super-capacitor, reinforcements in polymers and lithium and non-lithium-ion batteries [32-35].

Base fluid is an important element to prepare nanofluids. Silicone oil is one of the base fluids which has better heat transfer ability. Silicone oil is transparent, colorless, non-toxic, and has a 
121 broad viscosity range of 0.65-1 million cSt depending on the molecular weight and structure [36].

122 It is a polymer that contains silicon and oxygen atoms that produced artificially by composing 123 siloxane bonds silicone [36]. Generally, silicone oil can withstand high heat, high shear, and 124 corrosion because of the strong siloxane bonding [37]. Silicone oil can be used in higher 125 temperature (i.e. up to $400{ }^{\circ} \mathrm{C}$ ) continuously without changes in its property due to the heat 126 resistance. However, it has very low thermal conductivity which limits heat transfer performance 127 improvement.

Recently, nanofluids getting intensive interests in thermal applications due to their high thermo129 physical properties and added functional properties of base fluids. Silicone oil-based nanofluids have the high operating temperatures up to almost $400{ }^{\circ} \mathrm{C}$ which allows it to medium-to-high temperature applications like a solar thermal collector or concentrated solar power system. Silicone oil-based nanofluids can produce higher energy conversion efficiency as the solar thermal efficiency is proportional to the temperature rise of nanofluid. Although silicone oil-based fluid could perform better for solar thermal application, a stable dispersion of nanoparticles in the base fluid at relatively high operating temperatures has not been reported yet.

The dispersion of MXene nanoparticle into silicone oil to develop a stable nanofluid is the most challenging part of the MXene based nanofluid preparation. The MXene nanoparticles need to be suspended into the oil-based fluid to form a homogeneous nanofluid. Even though the stability of the nanofluid remains a challenge, silicone oil is stable at high-temperature (i.e. up to $400{ }^{\circ} \mathrm{C}$ ) [38]. Silicone oil cannot fulfill the requirement for the higher heat transfer and heat transfer improvement in special conditions. To ensure this issue, the nanofluid with the mixture of MXene nanoparticles and silicone oil could be a good option. Therefore, thermal conductivity, viscosity, TGA, FTIR, UV-Vis, and morphology have been studied to investigate the suitability of this material in CPVT applications. After the thermal properties study, MXene with silicone oil nanofluid is used in a Concentrated Photovoltaic Thermal system to investigate its thermal and electrical performance. Novelties of the present study are highlighted below:

147 We formulate new nanofluid with MXene and silicone oil for the first time. Thermal conductivity, 148 viscosity, thermal degradation of MXene based silicone oil at higher temperatures is the first study to the best of authors' knowledge. The electrical and thermal performance of this new fluid is also another new finding. Moreover, thermal conductivity found to be improved by $64 \%$ for the highest 
concentration. Besides, the viscosity of MXene with silicone oil nanofluids remains unchanged even with the additions of MXene nanoparticles in the silicon oil base fluid. This is another outstanding finding in relation to the viscosity performance. New correlations for the viscosity and thermal conductivity as a function of temperature and concentration are developed using experimental data. Findings on electrical and thermal performances in the CPVT system are also new outcomes.

\section{Methodology}

In the present research work, MXene $\left(\mathrm{Ti}_{3} \mathrm{C}_{2}\right)$ nanoparticles are synthesized using a wet chemistry method. Because of high viscosity of the silicone oil, a new method of preparation of MXene based silicone oil is developed. Four different solvents consisting of n-Hexane, chloroform, toluene and tween 40 are evaluated to obtain the uniform and less viscous solution. The mixture of silicone oil and the examined solvents (Toluene) are kept in the ratio of 1:1 (60 $\mathrm{ml}$ ). The mixture is stirred with $400 \mathrm{rpm}$ at $50{ }^{\circ} \mathrm{C}$ for 30 minutes. The same protocol is used for $\mathrm{n}-$ Hexane, chloroform and Tween 40. The resultant products reveal better dilution for utilized Toluene. Adding MXene nanoparticles to silicone oil is carried out with the dilution method by Toluene. Three different concentrations including 0.05, 0.08 and $0.1 \mathrm{wt} . \%$ of MXene nanoparticles are suspended in silicone oil to prepare samples. Thermo-physical properties consisting of thermal conductivity, thermal stability and viscosity are measured using THB 500, TGA and Rheometer, respectively. Optical properties and chemical structure are evaluated using UV-Vis and FTIR. Morphology of the synthesized MXene nanoparticles is studied using FESEM. The performance of the resultant nanofluid is assessed in a concentrated photovoltaic thermal collector.

\subsection{Preparation of MXene with silicone oil}

\subsubsection{Silicone oil, Toluene, Chloroform, n-Hexane and Tween 40}

Base fluid silicone oil is procured from R\&M Chemicals with viscosity of $350 \mathrm{~mm}^{2} . \mathrm{s}^{-}$

${ }^{1}$ at $25^{\circ} \mathrm{C}$. Toluene (Analytical reagent grade), Chloroform (Analytical reagent grade $=>99.8 \%$ ), n-Hexane (for analysis) and Tween 40 are obtained from Fisher Chemicals Company. 
Emerging nano-materials MXene $\left(\mathrm{Ti}_{3} \mathrm{C}_{2}\right)$ is synthesized using hydrofluoric acid (HF, 48\%

181

182

183

184

185

186

187

188

189

190

191

192

193

194

195

196

197

198

199

200

201

202

203

204

205

206

207

208 Fisher Chemical) and MAX phase powder $\left(\mathrm{Ti}_{3} \mathrm{AlC}_{2}\right)$. Following steps are used to synthesize MXene. MAX phase, $\mathrm{Ti}_{3} \mathrm{AlC}_{2}$ powder is immersed and stirred in hydrofluoric solution for 20 hours in a fume hood. The resultant mixture is centrifuged at $5000 \mathrm{rpm}$ for 5 minutes. Washing process of the mixture is then performed for several times to obtain $\mathrm{pH}$ above 5. The mixture is rinsed using methanol for 3 times and placed in an ultrasonic cleaner to remove contaminant from the mixture. Finally, the resultant MXene $\left(\mathrm{Ti}_{3} \mathrm{C}_{2}\right)$ is dried in a vacuum oven $(101 \mathrm{~L}$ VO500 MEMMERT) at $50{ }^{\circ} \mathrm{C}$ for 24 hours.

\subsubsection{Preparation of MXene with silicone oil as a new class of heat transfer fluids}

Preparation of the silicone oil-based MXene with three different concentrations $(0.05,0.08$ and $0.1 \mathrm{wt} . \%$ ) is processed in a precise protocol. Because of high viscosity of silicone oil, a new method of adding MXene is developed. Firstly, silicone oil is diluted in solvents to suspend in nanoparticles. For the purpose of diluting silicone oil, aromatic (ring structure) solvents such as toluene, xylene and naphtha can be affective. Furthermore, solubility of the silicone oil in chlorinated solvents such as trichloroethylene, perchloroethylene and methylene chloride is reported. Silicone oil is highly soluble in hydrocarbon solvents such as ligroin and mineral spirits. However, it is insoluble in ethanol, methanol and water. In this research work, 4 different solvents consisting of n-Hexane, chloroform, toluene and tween 40 are used to dilute the proposed silicone oil. The dilution process is performed with ratio of 1:1 for all 4 types of solvents. First of all, 60 $\mathrm{ml}$ of silicone oil (boiling point $>350{ }^{\circ} \mathrm{C}$ ) is added to a $150 \mathrm{ml}$ beaker, followed by the addition of $60 \mathrm{ml}$ of Toluene to the beaker. Then, the mixture is stirred with $400 \mathrm{rpm}$ at $50{ }^{\circ} \mathrm{C}$ for 30 minutes using a hot plate (RCT BASIC, IKA). The same protocol is used for n-Hexane, chloroform and Tween 40. The resultant products provide better dilution for utilized Toluene. Adding MXene nanoparticles to silicone oil is conducted with the diluted method by Toluene. For the purpose of preparing MXene based silicone oil with the concentration of $0.05 \mathrm{wt} . \%, 27 \mathrm{mg}$ of MXene is added to $53.973 \mathrm{~g}$ of diluted silicone oil.

The resultant fluid is sonicated for 30 minutes using an ultrasonic probe sonicator (FS-1200N) with power of $70 \%$. Same protocol is used for preparing MXene based silicone oil with loading concentrations of 0.08 and 0.1 wt.\%. MXene based silicone oil with concentrations of 0.08 and 
$0.1 \mathrm{wt} . \%$ is prepared with adding 43 and $54 \mathrm{mg}$ of MXene nanoparticles to 53.957 and $53.946 \mathrm{~g}$ of diluted silicone oil, respectively. The mixing procedure of silicone oil and MXene using

211 Toluene as a dilutor is accomplished in good condition. Evaporation procedure of the Toluene was

212 followed by setting the temperature of a hot plate at $120{ }^{\circ} \mathrm{C}$ (boiling point of toluene is $110{ }^{\circ} \mathrm{C}$ )

213 and stirring at $500 \mathrm{rpm}$ for 30 minutes to achieve the initial volume of silicone oil without any

214 solvent $(60 \mathrm{ml})$. The visual inspection of the prepared MXene based silicone oil with three different 215 concentrations including the base silicone oil is conducted for two weeks continuously. Figure 1 216 shows the condition of the prepared samples after two weeks. The UV-Vis spectroscopy analysis 217 reveals good stability for all prepared nanofluids in agreement with the visual inspection. Figure 2 218 presents experimental data for absorbance degradation analysis as a function of time (two weeks).

219 Monitoring the changes of the absorbance of the prepared MXene based silicone oil nanofluid 220 samples is another way to assess their stability. The absorbance spectra measurement is conducted 221 daily for 14 days to evaluate the absorbance degradation of the samples as illustrated in Figure 2. 222 Experimental spectra for three samples demonstrates slight variation of the absorbance spectra of 223 the prepared nanofluids. The experimentally acquired results for MXene based silicone oil with 224 loading concentration of $0.5 \mathrm{wt}$ \% indicate good stability of the prepared nanofluids. The acquired 225 absorbance for 14 days represent almost same trend with negligible change which proves good 226 stability of the nanofluids with less sedimentation. Same trend is observed for MXene based 227 silicone oil nanofluids with loading concentrations of 0.8 and $1 \mathrm{wt} \%$, which further proves good 228 stability of the suspended MXene nanoparticles in the silicone oil. This might be due to the high 229 specific surface area of MXene nanoparticles which improves the interface interaction between 230 MXene nanoparticles and silicone oil. 


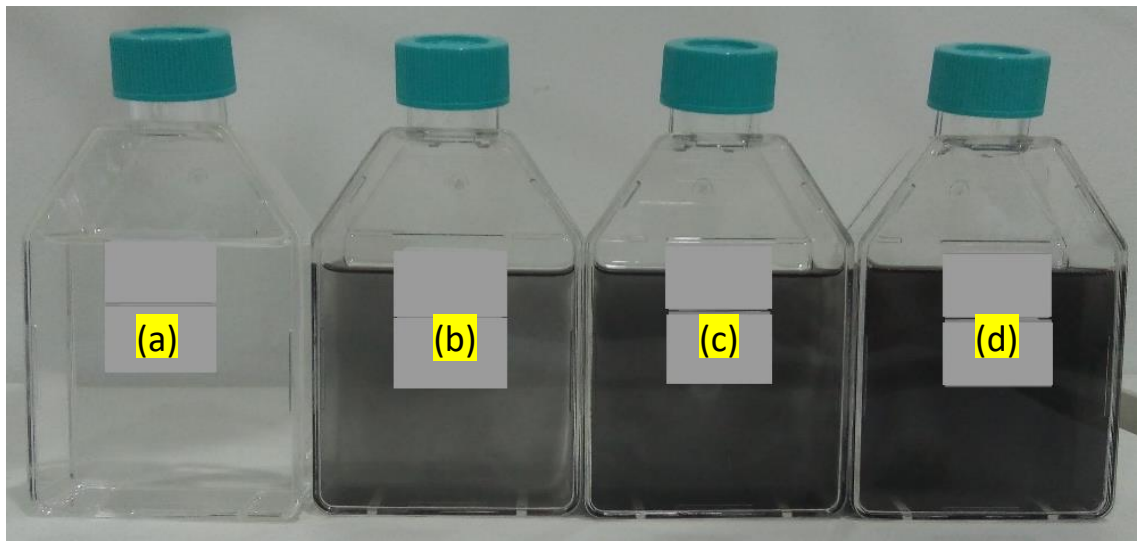

234 Figure 1. Prepared MXene based silicon oil in three different concentrations a) pure silicone oil, 235 b) 0.05 wt. $\%$, c) 0.08 wt. $\%$ and d) 0.1 wt. $\%$

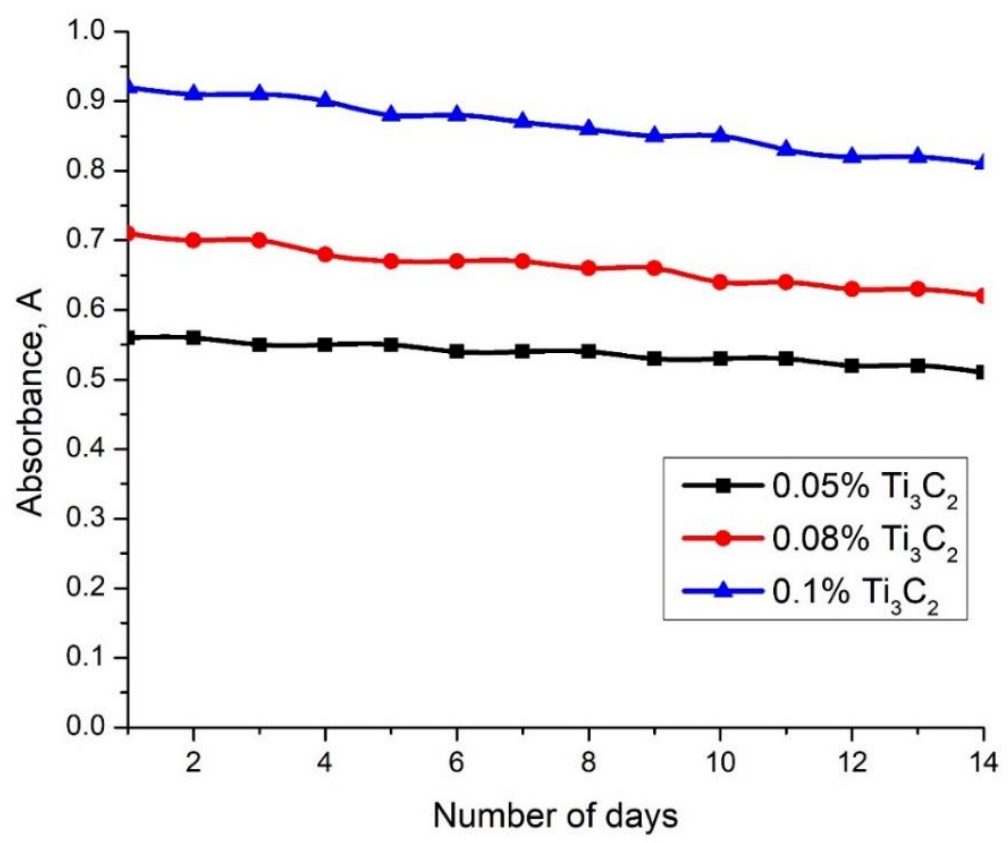

Figure 2. Absorbance degradation analysis of the prepared MXene based silicone oil nanofluids in three different concentrations as a function of time (14 days) 


\subsection{Thermo-Physical properties}

247

248

249

250

251

252

253

254

255

256

257

258

259

260

261

262

263

264

265

266

267

268

269

270

271

272

\subsubsection{Thermal Conductivity Measurement}

The thermal conductivity of MXene based silicone oil with different concentrations are measured using a Transient Hot Bridge (THB) 500 from Linseis (Germany) with a heater power $18 \mathrm{~mW}$ and current $5 \mathrm{~mA}$. For the purpose of stabilizing the sample before measurement, a waiting time of 15 seconds is used. THB has broad range of thermal conductivity ( 0.01 to $500 \mathrm{~W} / \mathrm{m} . \mathrm{K}$ ) with high accuracy due to the patented sensor design and covers broad range of temperature (-150 to $700{ }^{\circ} \mathrm{C}$ ). In this research work, thermal conductivity measurements are performed using Linseis Hot Point Sensors (HPS). The HPS sensors work according to the transient plane method and suitable to measure anisotropic samples. Due to the small amount of heat, which is produced by the hot point sensors, it is a good choice to measure liquids with negligible convection. The principle of the THB 500 is based on newly developed Quasi-Steady-State (QSS) method for the measurement of thermal conductivity. The temperature dependency measurements are performed for temperatures including $25,50,100$ and $150{ }^{\circ} \mathrm{C}$. The temperature is maintained using a hot plate (RCT BASIC, IKA).

\subsubsection{Viscosity measurement of MXene based silicone oil}

The viscosity measurement is performed using a Rheometer (Anton Paar model MCR92). The share rate measurement as a function of temperature is conducted for all samples $(60 \mathrm{ml})$ for 5 temperatures $25,50,75,100$ and $125^{\circ} \mathrm{C}$. Viscosity measurement for the temperature $150{ }^{\circ} \mathrm{C}$ cannot be measured due to the limitation of the equipment used. T-Ramp measurement (viscosity as a function of temperature) is performed for the pure silicone oil and MXene based nanoparticles with silicone oil in different concentrations consisting of $0.05,0.08$ and $0.1 \mathrm{wt} . \%$.

\subsubsection{Thermal stability test using TGA}

Thermogravimetric analysis (TGA) of the pure silicone oil and silicone oil-based MXene has been conducted using Perkin Elmer TGA 4000. A $180 \mu$ l alumina crucible that can withstand $1750{ }^{\circ} \mathrm{C}$ under an ultra-high pure nitrogen gas flow of $19.8 \mathrm{ml} / \mathrm{min}$ with the gas pressure of $2.6 \mathrm{bar}$ is selected to examine the samples. The utilized heating rate was $10^{\circ} \mathrm{C} / \mathrm{min}$ to raise the temperature 
273 from 30 to $800{ }^{\circ} \mathrm{C}$. About $10 \mathrm{mg}$ of pure silicone oil is used for the decomposition temperature

274 measurement. Decomposition temperature measurement for the other samples is followed with 275 same protocol (10 mg). The obtained data is analyzed using Pyris Software.

2.3.1 Fourier Transform Infrared Spectroscopy (FTIR) analysis

Perkin Elmer Spectrum Two-UATR spectra with integrated detector of MIR TGS (15000-370 $\mathrm{cm}^{-1}$ ) is used to detect the peak and the functional group of silicone oil and different concentration of $\mathrm{Ti}_{3} \mathrm{C}_{2}$ dispersed into silicone oil. The scanning speed used to detect the Fourier Transform Infrared Spectrum (FTIR) of the silicone oil and the nanofluids is maintained constant $0.2 \mathrm{~cm} / \mathrm{s}$ with the optimum scan range of $4000-450 \mathrm{~cm}^{-1}$.

\subsubsection{UV-Vis analysis of the silicone oil-based MXene}

Perkin Elmer Lambda 750 is used to perform Ultraviolet-visible spectroscopy (UV-Vis) to get 285 the optical absorbance. The absorption data is collected at room temperature with the wavelength range from 800 to $200 \mathrm{~nm}$. The adjusted scan speed is $266.75 \mathrm{~nm} / \mathrm{min}$ with the $860 \mathrm{~nm}$ monochromatic.

\subsubsection{Morphology and microstructure analysis of MXene nanoparticles}

Morphology of the MXene flakes is investigated using FESEM (Hitachi SU8000) imaging at an accelerating voltage of $15 \mathrm{kV}$. Working distance is set to be $15,900 \mu \mathrm{m}$ with emission current of 10,500 nA. Microstructure of the MXene flakes is investigated using HRTEM (JEOL JEMARM 200F) imaging at an accelerating voltage of $200 \mathrm{kV}$. About $1 \mathrm{mg}$ of MXene flake is added into $\sim 4 \mathrm{ml}$ of ethanol in a vial. The mixture is kept on the hot plate at $60{ }^{\circ} \mathrm{C}$ and stirred using a magnetic stirrer for half an hour. About $4 \mu \mathrm{l}$ of diluted sample is then taken by a micropipette and dripped onto the carbon-coated cupper grid. An energy dispersive X-ray spectroscopy (EDX, Oxford instrument) is used to confirm the elemental map imaging and observation of spatial distribution of all the elements in both FESEM and HRTEM analysis. The spot analysis is carried 
301

302

303

304

305

306

307

308

309

310

311

312

313

314

315

316

317

\subsubsection{CPVT Collector System Configuration}

In a typical CPVT system, the cooling fluid is used to cool the PV cells to avoid overheating. The cooling fluid channel is placed beneath the PV module. Sidewalls of the channel are insulated. the upper surface of the channel is exposed to the PV module. Figure 3 shows a schematic diagram of a CPVT collector system. The MXene nanoflakes are dispersed in the cooling fluid to remove excess heat from the PV panel.

The PV panel is directly exposed to the incident radiation and it converts less than $20 \%$ of the energy to electric power. Most of the remaining energy is absorbed by the PV panel and raises PV surface temperature. This is undesirable as electrical efficiency will be lowered due to higher PV surface temperature. Heat transfer to the cooling fluid channel from the upper surface of the PV panel is by convection and radiation from the sky, while the heat transfer from the bottom surface is through convection and radiation from the ground. The enhancement of the thermal conductivity of the cooling fluid by the inclusion of potential emerging nanomaterials, MXene expected to improve the cooling of the panel. Hence, the effect of the loading of MXene nanoparticles on the performance of the system is studied under different weather conditions (atmospheric temperature and concentration of solar radiation) and at different operating conditions (channel height and flow rate of the cooling fluid). 


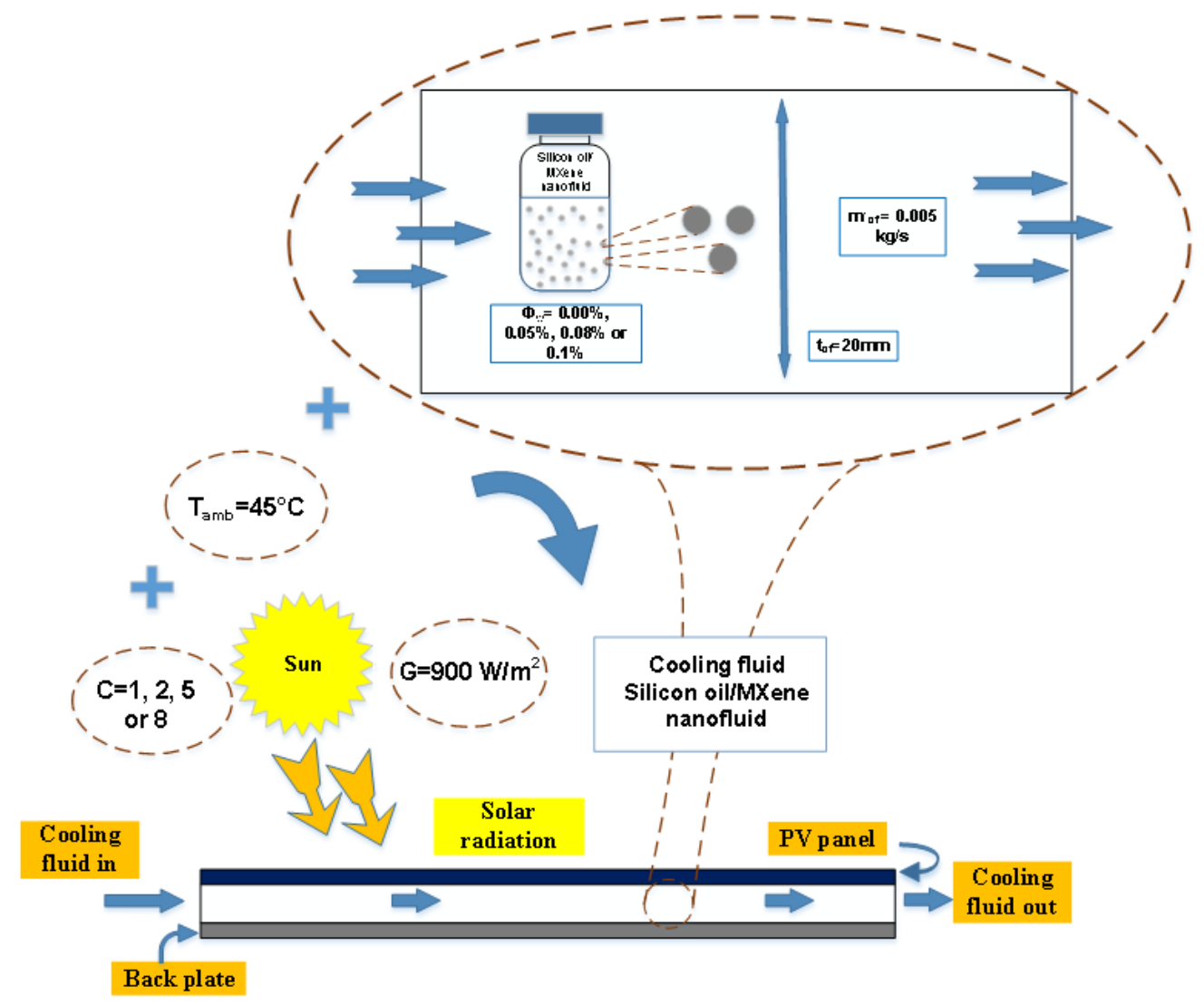

Figure 3. Schematic diagram for a typical CPVT system

\subsubsection{Numerical calculation of thermo-physical properties}

Due to the limitations in the measurement facilities, the specific heat of the silicon oil/MXene nanofluid is calculated using the formulas commonly used in studies published in the literature [39] as follows:

$$
\left(\rho c_{p}\right)_{c f}=\varnothing\left(\rho c_{p}\right)_{n p}+(1-\varnothing)\left(\rho c_{p}\right)_{f}
$$

where, $c_{p_{n p}}, c_{p_{f}}$ and $c_{p_{c f}}$ are the specific heat of the nanoparticles, the base fluid, and the nanofluid, respectively, in J/kg.K. The dependence of the MXene nanoparticles specific heat on the temperature is measured experimentally and correlated as follows:

$$
c_{p_{n p}}=-0.01 T_{c f}^{2}+3.4 T_{c f}+604.5
$$

Where, $T_{c f}$ is in $\left({ }^{\circ} \mathrm{C}\right)$

1 Due to the difficulty in the measurements of the density and the unknown value of the MXene

32 nanoparticles density, the density of the silicon oil/MXene is assumed to be constant and equal to 
333 the density of the pure silicon oil. Indeed, the effect of the nanoparticles loading on the density of 334 the nanofluid could be effective at high loadings values. Neglecting the effect of the density 335 change as nanoparticles loadings are very small, the specific heat of the silicon oil/MXene 336 nanofluid is calculated as follows:

$337 c_{p_{c f}} \cong \emptyset c_{p_{n p}}+(1-\emptyset) c_{p_{f}}$

338

339

\subsection{Thermal and electrical models}

Mathematical models of the CPVT have been developed to study the performance of the system numerically. Transient two-dimensional governing mathematical equations are developed, and the model has been divided into two parts, i.e., the thermal model and the power generation model. Matlab 2017b software program was used to analyze the data.

\subsubsection{Thermal model}

Heat transfer in a typical CPVT system normally occurs through conduction, convection, and radiation. A portion of the incident solar energy is lost by radiation and convection to the atmosphere, a portion is absorbed by the PV panel, and a portion is converted to electricity. The absorbed part is transferred through the lower surface of the panel to the cooling fluid channel beneath the PV module by conduction and convection.

The energy balance equations along the main parts of the CPVT system are presented as follows:

PV panel:

$m_{p v} c p_{p v} \frac{d T_{p v}}{d t}=\alpha_{\mathrm{pv}} \Delta x W C G-\Delta x W C G \eta_{r e f}\left[1-\beta_{p v}\left(T_{p v}-T_{r e f}\right)\right]+\Delta x W h_{r a d, \mathrm{pv}}\left(T_{s k y}-\right.$

$\left.T_{\mathrm{pv}}\right)+\Delta x W h_{\mathrm{conv}, p v}\left(T_{a m b}-T_{\mathrm{pv}}\right)+\Delta x W h_{c o n v, c f}\left(\mathrm{~T}_{\mathrm{cf}}-\mathrm{T}_{\mathrm{pv}}\right)+\frac{W t_{p v} k_{p v}}{\Delta x}\left(T_{\mathrm{pv}_{i+1}}-T_{\mathrm{pv}}\right)+$ $\frac{W t_{p v} k_{p v}}{\Delta x}\left(T_{\mathrm{pv}_{i-1}}-T_{\mathrm{pv}}\right)$

Eq. 4

Cooling fluid:

$m_{c f} c p_{c f}\left(T_{\text {cf,out }}-T_{\text {cf,in }}\right)=\Delta x W h_{c o n v, c f}\left(\mathrm{~T}_{\mathrm{pv}}-\mathrm{T}_{\mathrm{cf}}\right)+\Delta x W h_{c o n v, c f}\left(\mathrm{~T}_{\mathrm{bp}}-\mathrm{T}_{\mathrm{cf}}\right)$

Eq. 5

Back plate:

$$
m_{b p} c p_{b p} \frac{d T_{b p}}{d t}=\Delta x W h_{c o n v, c f}\left(\mathrm{~T}_{\mathrm{cf}}-\mathrm{T}_{\mathrm{bp}}\right)+\Delta x W h_{r a d, b p}\left(T_{\text {ground }}-T_{\mathrm{bp}}\right)+
$$


362 where, $T_{i+1}$ and $T_{\mathrm{i}-1}$ are the temperatures of the layer to the right and left directions on the x-axis 363 from the calculation point.

$364 h_{r a d, p v}$ and $h_{r a d, b p}$ are the radiation heat transfer coefficients at the upper and lower surfaces of 365 the system calculated using the following formulas described by Cengel and Ghajar [40]:

$h_{\text {rad }, \mathrm{pv}}=\Delta x W \varepsilon_{p v} \sigma\left(T_{s k y}+T_{\mathrm{pv}}\right)\left(T_{s k y}^{2}+T_{\mathrm{pv}}{ }^{2}\right)$

$368 h_{c o n v, p v}$ and $h_{c o n v, b s}$ are the convection heat transfer coefficients calculated at the upper and lower 369 surfaces of the system using the formulas mentioned in Cengel and Ghajar [40] and depends on 370 the type of convection as follows:

371 For natural convection:

372 PV panel (faces upward):

373

$h_{c o n v, p v}= \begin{cases}\left(\frac{k_{a}}{L}\right) 0.54 R a^{\frac{1}{4}} & T_{\mathrm{pv}}>T_{\mathrm{amb}} \\ \left(\frac{k_{a}}{L}\right) 0.27 R a^{\frac{1}{4}} & T_{\mathrm{pv}}<T_{\mathrm{amb}}\end{cases}$

374 where, $R a=\frac{g \beta_{f}\left(T_{p v}-T_{a m b}\right) L^{3}}{v_{a} \alpha_{a}}$

375 Back plate (faces downward):

$376 h_{c o n v, b p}= \begin{cases}\left(\frac{k_{a}}{L}\right) 0.27 R a^{\frac{1}{4}} & T_{\mathrm{bp}}>T_{\mathrm{amb}} \\ \left(\frac{k_{a}}{L}\right) 0.54 R a^{\frac{1}{4}} & T_{\mathrm{bp}}<T_{\mathrm{amb}}\end{cases}$

377 For forced convection:

$378 \quad h_{\text {conv }}=\left(\frac{k_{a}}{L}\right) 0.664 \operatorname{Re}_{a}^{\frac{1}{2}} \operatorname{Pr}_{a^{\frac{1}{3}}}$

Eq. 11

379 where, $R e_{a}=\frac{u_{a} L}{v_{a}}$

$380 h_{c o n v, c f}$ is the convection heat transfer coefficient of the cooling fluid and can be calculated using 381 the following formulas, which depend on the nature of the flow (i.e., developing or fully 382 developed) and the existence of a laminar flow.

$383 h_{c o n v, c f}=\frac{N u_{c f} k_{c f}}{D_{h}}$

Eq. 12 
$384 N u_{c f}=\left\{\begin{array}{cl}3.66+\frac{0.065 \operatorname{Re}_{c f} P r_{c f} \frac{D_{h}}{L}}{1+0.04\left(\operatorname{Re}_{c f} P r_{c f} \frac{D_{h}}{L}\right)^{\frac{2}{3}}} & 0.05 R e_{c f} t_{c f}>0.1 L \text { (Developing) } \\ 8.24 & 0.05 R e_{c f} t_{c f}<0.1 L \text { (Fully developed) }\end{array}\right.$

\subsubsection{Power generation model}

The electrical power generated by the CPVT system is defined by Eq. 14 .

$$
P_{e l, o u t}=L W C G \eta_{\text {ref }}\left[1-\beta_{p v}\left(T_{p v}-T_{\text {ref }}\right)\right]
$$

The electrical efficiency and its relationship with the PV panel temperature is defined by Eq. 15.

$$
\eta_{e l}=\frac{P_{e l, o u t}}{P_{\text {in }}}=\eta_{\text {ref }}\left[1-\beta_{p v}\left(T_{p v}-T_{\text {ref }}\right)\right]
$$

where, $P_{\text {in }}$ is the input power, which is evaluated as: $P_{\text {in }}=L W C G$

The reference efficiency $\left(\eta_{\text {ref }}\right)$ and the temperature coefficient $\left(\beta_{p v}\right)$ of the CPV are considered constants at $20 \%$ and $0.005 /{ }^{\circ} \mathrm{C}$, respectively.

The heat collected by the cooling fluid in the CPVT system and the resulting thermal efficiency of the system are represented by Eq. 16 and 17.

$$
\begin{gathered}
Q_{t h}=m_{c f} c p_{c f}\left(T_{\mathrm{cf}, \mathrm{out}}-T_{\mathrm{cf}, \mathrm{in}}\right) \\
\eta_{t h}=\frac{Q_{t h}}{P_{\text {in }}}
\end{gathered}
$$

The thermal and electrical output energies from the system can be calculated, depending on the study time as follows:

$E_{t h}=Q_{t h} \times \Delta$ Time

Eq. 18

$E_{\text {el }}=P_{\text {el,out }} \times \Delta$ Time

\subsubsection{Parametric Investigation}

The main target of developing this numerical solution is to assess the effect of the inclusion of the MXene nanoparticles in the silicone oil on the performance of the hybrid CPVT system. The performance of the CPVT is evaluated at the different nanoparticle loadings $(0.05,0.08$ and 0.1 wt.\%) and solar concentrations (1, 2, 5 and 8) using the mathematical model equations 4-6 and 1419. The temperature of the CPV panel, the electrical energy generated, and the electrical and thermal efficiencies are used as indicators of the performance of the system. The input parameters 
411 involved in the study are presented in Table 1.

412

413

Table 1. Input parameters used in the study

\begin{tabular}{cccc}
\hline Parameter & Value & Parameter & Value \\
\hline$L$ & $0.7 \mathrm{~m}$ & $T_{a m b}$ & $45^{\circ} \mathrm{C}$ \\
$W$ & $0.3 \mathrm{~m}$ & $G$ & $900 \mathrm{~W} / \mathrm{m}^{2}$ \\
$t_{p v}$ & $3 \mathrm{~mm}$ & $\Delta x$ & $0.05 \mathrm{~m}$ \\
$t_{b p}$ & $3 \mathrm{~mm}$ & $\Delta t$ & $10 \mathrm{sec}$ \\
$t_{c f}$ & $20 \mathrm{~mm}$ & $T_{r e f}$ & $25^{\circ} \mathrm{C}$ \\
$m_{c f}$ & $0.005 \mathrm{~kg} / \mathrm{s}$ & $u_{a}$ & $0 \mathrm{~m} \mathrm{~s}^{-1}$ \\
$\Delta$ Time & $3600 \mathrm{~s}$ & &
\end{tabular}

414

415

3. Results and Discussion

Experimentally measured thermal conductivities of MXene with silicone oil nanofluid samples 418 for the varying loading of MXene nanoflakes are plotted in Figure 4 as a function of temperature.

419 The effect of temperature on the thermal conductivity of MXene with silicone oil nanofluids is 420 analyzed. Y-axis error bars with the experimental thermal conductivity values are the standard 421 deviations of the measured thermal conductivity of the nanofluids. Standard deviations are found 422 to be \pm 0.002 . It is seen in that thermal conductivity of MXene with silicone oil nanofluid samples 423 is increased linearly with the increasing temperature. From the analysis, it is found that the thermal 424 conductivity of MXene with silicone oil nanofluid is dependent on both temperature and increased 425 loading of MXene nanoflakes in silicone oil. MXene with silicone oil nanofluid sample with 0.1 426 wt.\% shows the highest thermal conductivity which is $\sim 0.203 \mathrm{~W} / \mathrm{m} \mathrm{K}$ at $25{ }^{\circ} \mathrm{C}$ and it reaches to $4270.263 \mathrm{~W} / \mathrm{m} \mathrm{K}$ at $150{ }^{\circ} \mathrm{C}$. This remarkable improvement is possible because of the presence of 428 extremely large basal plane of MXene sheets in silicone oil and high thermal conductivity through 429 the MXene nanoflakes basal plane [41, 42]. 


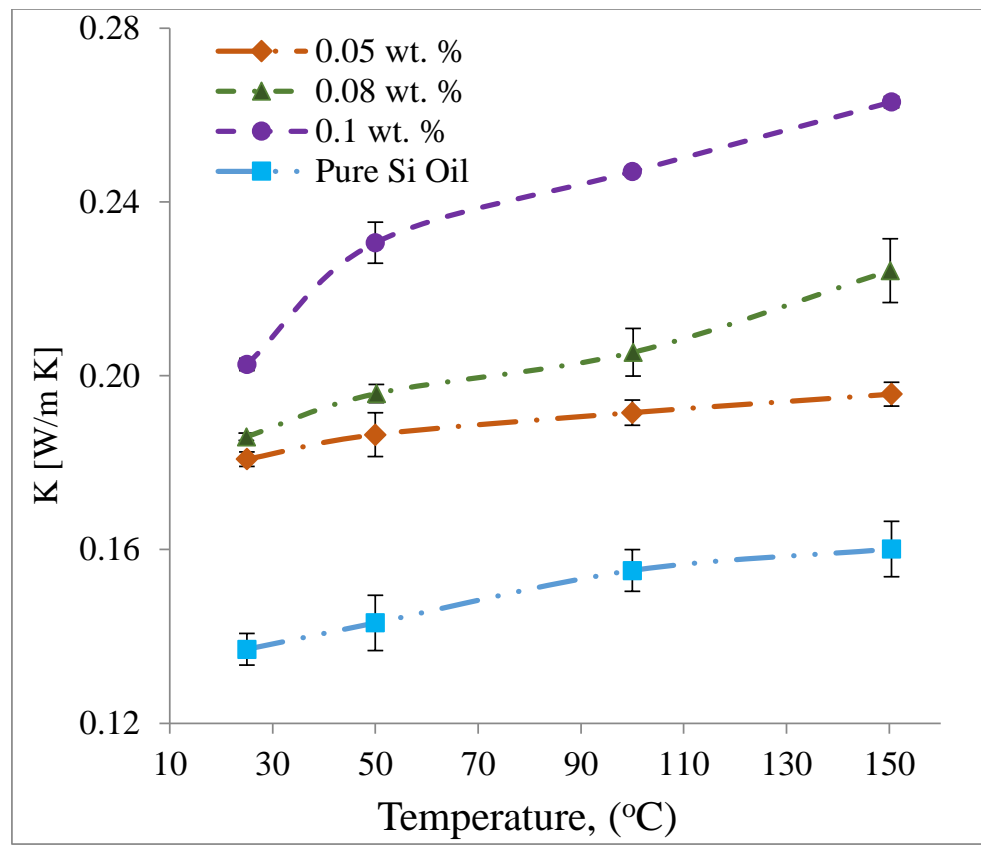

Figure 4. Thermal conductivity of MXene with silicone oil nanofluids as a function of temperature for the varying MXene nanoflakes loading of $0.05,0.08,0.1 \mathrm{wt} . \%$

Due to increase of PV cell temperature over the whole PV module by $10-15^{\circ} \mathrm{C}$, the electric power is decreased $\sim 6 \%$ in real operating conditions [43]. Surface temperature of the PV panel can be reduced significantly by removing excess heat from PV panel with the improvement of thermal conductivity of the heat transfer fluids [44]. Thus, PV panel temperature can be kept uniform by increasing the thermal conductivity of the cooling fluids used in the channel attached [43].

Figure 5 shows the overall percentage of thermal conductivity enhancements of MXene with silicone oil nanofluids with the MXene loading of 0.05, 0.08 and $0.1 \mathrm{wt}$. \% over silicone oil at four different temperatures varied from 25 to $150^{\circ} \mathrm{C}$. Percentage of thermal conductivity enhancements of MXene with silicone oil nanofluid samples are estimated using the correlation $\left(\left(\mathrm{K}_{\mathrm{nf}}{ }^{-}\right.\right.$ $\left.\left.\mathrm{K}_{\mathrm{f}} / \mathrm{Kf}\right)\right) \times 100 \%$. Here, $\mathrm{K}_{\mathrm{nf}}$ is the thermal conductivity of MXene with silicone oil nanofluids and $\mathrm{K}_{\mathrm{f}}$ is the thermal conductivity of base fluid (pure silicone oil). Figure 5 describes the effect of addition of MXene nanoflakes on the thermal conductivity enhancements of MXene with silicone oil nanofluids over the silicone oil at different temperature. It is seen that addition of MXene nanoflakes in silicone oil provides drastic enhancement of thermal conductivity over the silicone 
oil. Because of the contribution of high basal plane, thermal conductivity of MXene nanoflake is increased by both MXenes loading and temperature [45].

For a certain wt.\% of MXene loading, thermal conductivity enhancements are varied minimally with the rise in temperature. For the silicone oil with 0.05 wt.\% MXene loading, the enhancement of thermal conductivity is found to be varied about 10-15\% over silicone oil for the temperature range 25 to $150{ }^{\circ} \mathrm{C}$. Similar trends are observed for sample with 0.08 and 0.1 wt.\% loading of MXene nanoflakes. On the other hand, for a certain temperature, thermal conductivity of MXene with silicone oil nanofluids increased significantly with the increase of MXene loading in the silicone oil. At $150{ }^{\circ} \mathrm{C}$, percentage increase of thermal conductivity is obtained $\sim 22, \sim 40$ and $64 \%$ over silicone oil for the MXene loading of 0.05, 0.08 and $0.1 \mathrm{wt} . \%$, respectively. Similar trends are observed in the lower temperatures as well. It means, a lower degree of thermal conductivity enhancement is found for certain MXene loading with the increasing temperature. In contrast, for a certain temperature, higher enhancement of percentage of thermal conductivity over silicone oil is observed with the increasing loading of MXene in silicone oil. It reveals that, effect of MXene loading is higher than that of increasing temperature on the thermal conductivity enhancements of the MXene with silicone oil. Similar phenomenon was also perceived by Gu, Xie [46] when authors investigated the thermal conductivity of sheet like graphene flakes dispersed nanofluids.

Thus, the addition of MXene nanoflakes with silicone oil offers considerably high thermal conductivity. Thermal conductivity of MXene nanoflakes increases with the increase in temperature. So, the contribution of thermal conductivity also increases in MXene with silicone oil nanofluids. Moreover, smaller flakes (such as $<1 \mu \mathrm{m}$ ) could move randomly with the increasing temperature so that energy transport inside the base fluids becomes stronger [47, 48]. In this case, electron can hop from one flake to other in the nanofluid [41].

Thermal conductivity of the MXene nanoflakes suspended nanofluids deteriorate very negligibly by Brownian motion, possibly due to the flexibility and extra-large surface area of the 2D flakes like graphene [49]. However, at room temperature percentage of thermal conductivity enchantments are found lower than that of the higher temperatures. Because, in this temperature, there is no effect of Brownian motion of the particles [47, 48, 50]. This can be because of chain 
mechanisms created in the fluids influencing thermophoresis effect and Benard-Maragoni effect and also the inherent enhancements in the thermal conductivity of the nanofluid. These mechanisms always lead to the rise of the thermal performance of the system [51].

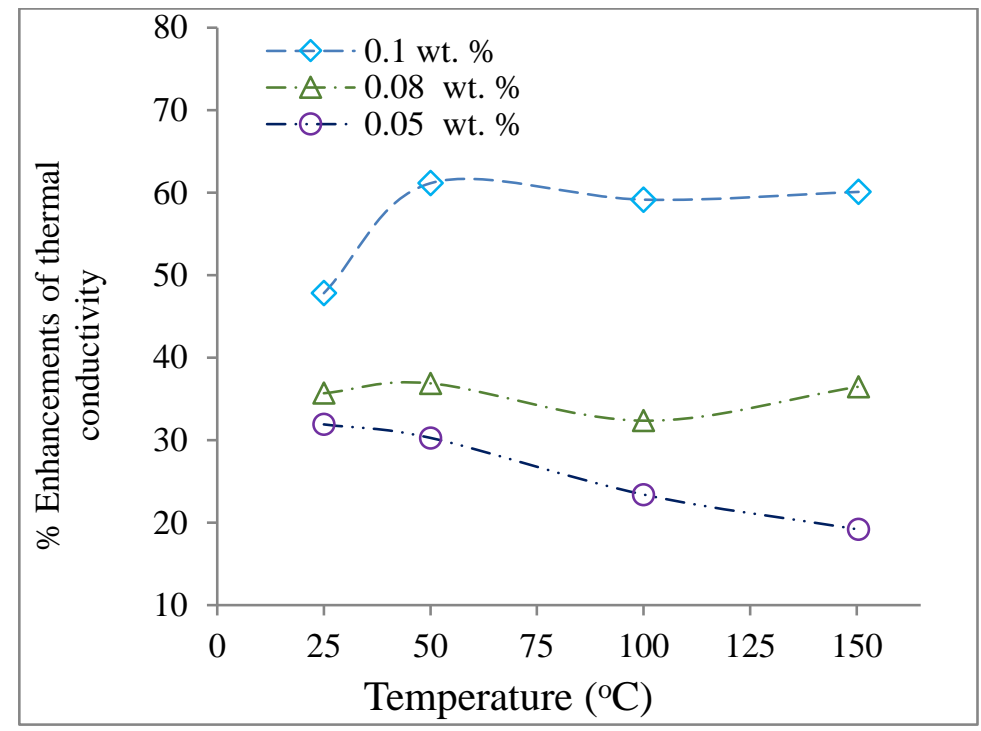

Figure 5. Percentage of thermal conductivity enhancements of MXene with silicone oil nanofluids as a function of temperature for different concentrations

\subsection{Viscosity of MXene with Silicone Oil Nanofluids}

To evaluate the rheological behaviors of silicon oil/MXene nanofluids, the viscosity is measured with varying shear rate at five different temperature ranges from 25 to $125{ }^{\circ} \mathrm{C}$. The variationsof the viscosity with silicone oil and MXene nanofluids with varying wt.\% as a function of shear rate is plotted in Figure 6.

, The viscosity of MXene with silicone oil nanofluids does not change as a function of shear rate within the range investigated at a certain temperature. It indicates that MXene with silicone oil nanofluids behaves as a Newtonian fluid. This phenomenon is also perceived for every temperature difference. Newtonian shear might be dueto the spindle rotation and the alignments of the fluid molecules those are not decorated [52]. If the shear rate is increased further, it will not make any difference to the viscosity. This means that MXene with the viscosity of silicone oil 
nanofluids is independent of the rate of shear force. Silicone oil is normally comprised of small isotropic (symmetric in shape and properties) molecules that are not oriented by the flow.

501 On the other hand, viscosity of MXene with silicone oil nanofluid samples only depends on 502 temperature. MXene with silicone oil nanofluids show the higher viscosity at lower temperature 503 504 the viscosity remains constant.

505
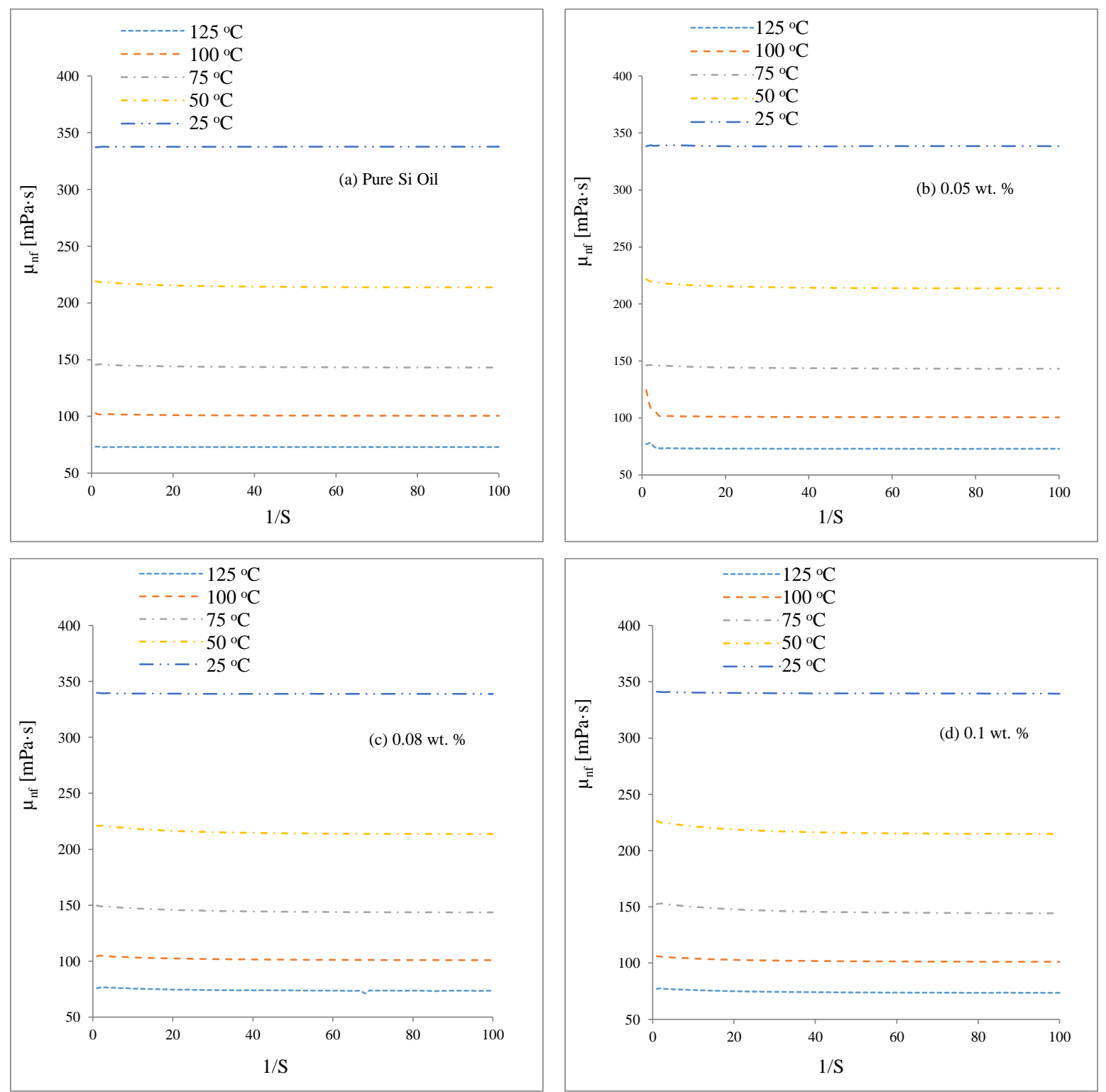

Figure 6. Kinematic viscosity of MXene with silicone oil nanofluids as a function of shear rate at different temperatures: (a) Pure silicon oil, (b) 0.05, (c) 0.08, (d) 0.1 wt.\% 
Typically increase of particle loading in the fluids incur the increase of viscosity [53]. Increasing

511 viscosity adversely effects the heat transport properties of the nanofluids [54]. Increase of viscosity

512 of the heat transfer nanofluids resulting in increase of erosion of carrying channels, pumps and

513 heat exchangers [53]. Due to the implication of nanoflakes (MXene), the effect of momentum and

514 the kinetic energy will be very less on the solid surfaces of the carrying channels which leads to

515 the reduction of erosion and pump efficiency used in the CPVT system.

516 The viscosity of MXene with silicone oil nanofluids at a high shear rate of $100 \mathrm{~s}^{-1}$ at different

517 wt.\% for all tested temperatures is reported in Figure 7. Surprisingly, it can be seen that, at a certain

518 temperature, viscosity of MXene with silicone oil nanofluids is not changed with the increasing

519 loading of MXene nanoflakes in the silicone oil base fluid. It means that viscosity of silicone oil

520 is independent of MXene additions. MXene nanoflakes act as large anisotropic molecules. For this

521 reason, when MXene nanoflakes are added in the silicone oil in low concentrations, MXene with

522 silicone oil nanofluids display a constant viscosity regardless of shear rate. While viscosity of

523 MXene with silicone oil nanofluids and silicone oil base fluid are strongly dependent on

524 temperature, it is also observed that the viscosity is decreased at higher temperatures. This

525 temperature effect on viscosity is related to the weakening of the inter particle and inter molecular

526 adhesion forces. It can be further explained as, with the increase of fluid temperature, the average

527 speed of the molecules increases and the amount of time they spend in contact with their nearest

528 neighbors' decreases. Thus, with the increase in temperature, the average intermolecular forces

529 decrease which, in turn, reduces the viscosity [52]. Notably it is seen that viscosity is reduced about

$53037 \%$ for the increase of temperature by $\sim 25^{\circ} \mathrm{C}$ for all the MXene concentrations with silicone oil

531 nanofluids samples. This percentage reduction of viscosity is about $\sim 27 \%$ at $125^{\circ} \mathrm{C}$ with the

532 increase of temperature by $25^{\circ} \mathrm{C}$ (as in Figure 7). 


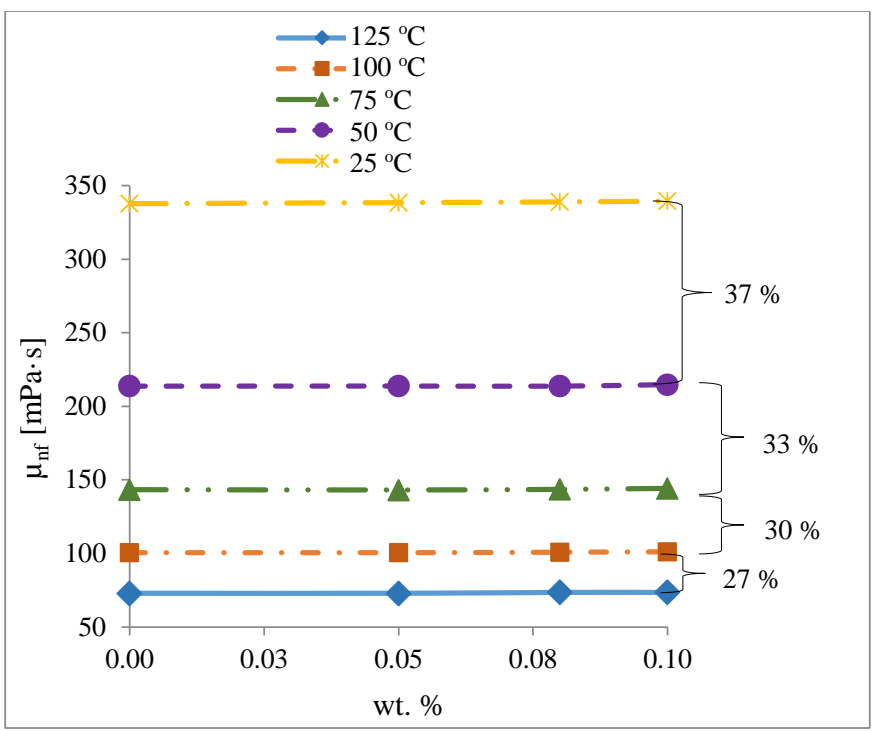

Figure 7. Viscosity of MXene with silicone oil nanofluids samples as function of MXene loading in the silicone oil base fluids

\subsection{Thermal Stability of silicone oil-based MXene}

Thermal durability of the pure silicone oil and silicone oil-based MXene in different concentrations is evaluated by thermogravimetric analysis (TGA) and presented in Figure 8. TGA measurement is performed with mass value of $10 \mathrm{mg}$ and heating rate of $10{ }^{\circ} \mathrm{C} / \mathrm{min}$ in the temperature range $30-900{ }^{\circ} \mathrm{C}$ to ensure the accuracy of the obtained results. It is revealed that the initial and final degradation temperature of pure silicone oil increases with the increase in the concentration of MXene nanoparticles. This research work indicates that adding MXene nanoparticles increases thermal stability of commercially available silicone oil. Enhancement of $9.8 \%$ is achieved for the highest loading of MXene nanoparticles ( $0.1 \mathrm{wt} . \%)$ compared with pure silicone oil. The onset degradation temperatures for pure silicone oil and silicone oil-based MXene nanofluids consisting of three different concentrations are $346,358,367$ and $380{ }^{\circ} \mathrm{C}$. The decomposition temperature for pure silicone oil is close to the commercially available silicone oil [55]. Silicone oil-based MXene nanofluids show 3.5 and $6.2 \%$ enhancement for concentrations of 0.05 and 0.08 wt.\%, respectively. From the Figure 8, it can be observed that with adding MXene nanoparticles, the degradation step becomes smoothed compared with pure silicone oil. According to the Thermo-gravimetric analysis (Figure 8), the IDT (initial decomposition temperature) of the silicone oil-based MXene with concentration of $0.08 \mathrm{wt} \%$ is found higher than loading 


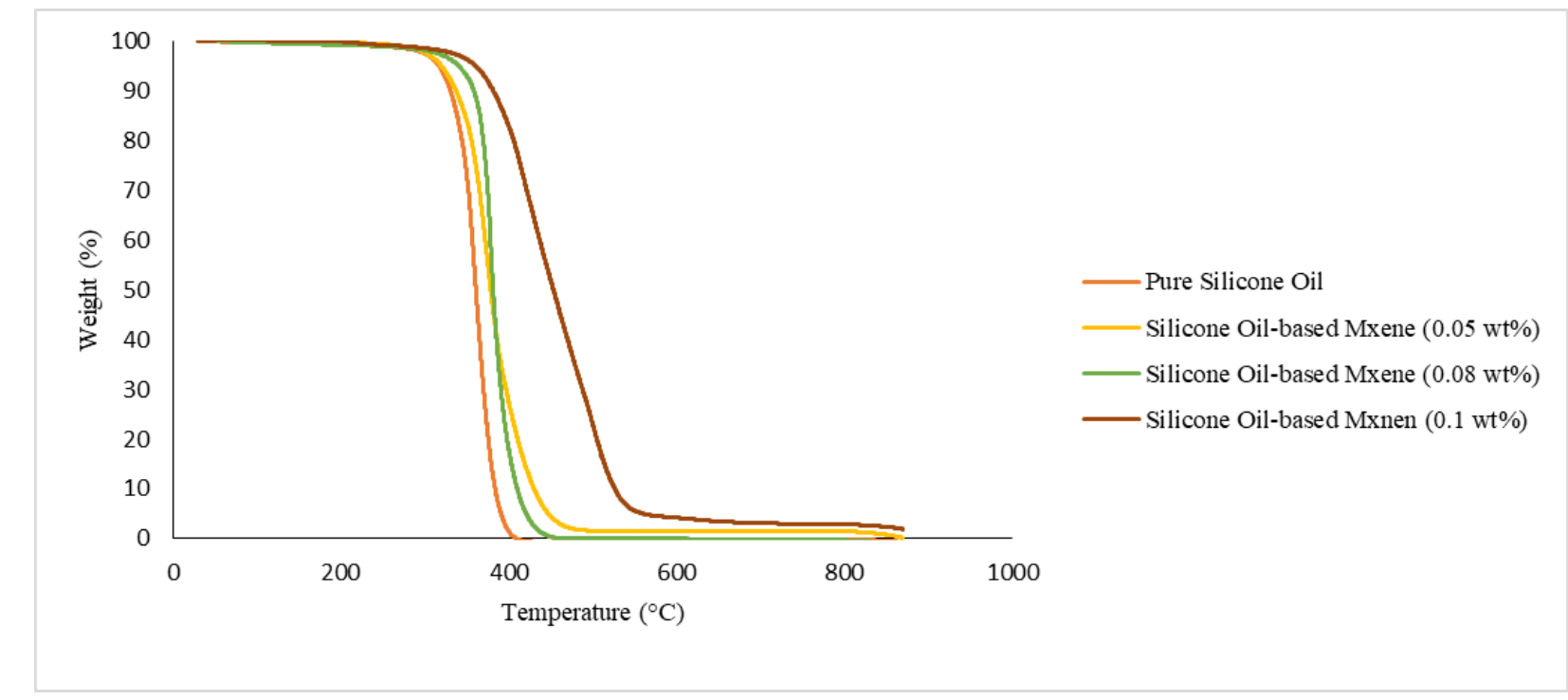
residue) of the silicone oil-based MXene with loading concentration of $0.05 \mathrm{wt} . \%$ becomes higher than the loading concentration of $0.08 \mathrm{wt} . \%$. The increment in D1/2 and FR of the silicone oilbased MXene with loading concentration of $0.5 \mathrm{wt} . \%$ in comparison with loading concentration of 0.8 wt.\% might be due the activation energy in temperature above $400{ }^{\circ} \mathrm{C}$. Since MXene nanomaterial has very high SSA (specific surface area), the interface interactions between MXene nanomaterial and silicone oil in higher temperature might increase the activation energy which is directly related with the decomposition temperature [56].

Silicone oils are used as lubricants at high operating temperatures for applications depending on rolling friction [57]. The fluids are also used in solar collectors, shock absorbers, hydraulic fluids, dashpots and other damping systems designed for high-temperature operation. Due to the beneficial advantages of silicone oils in high temperatures, the critical point (thermal stability) is an important parameter. Conventional silicone oils have been used in plastics processing for a long time as internal and external lubricants. The usage of silicone oils has caused enormous advantages in terms of texture, strength, pliability and special finishes. Their superior lubrications properties leads to enhance the productivity [58]. Thermal stability of lubricants at high temperatures is one of the crucial criteria which should be taken into account carefully. The interface interaction between silicone oil and MXene nanoparticles, due to the high surface area of MXene layers might be the reason of enhancement in thermal durability of silicone oil-based MXene. 
Figure 8. TGA results of the silicone oil-based MXene nanofluids

Figure 9 shows the FTIR spectrum of silicone oil and different concentration of MXene and silicone oil for the frequency range of $4000-450 \mathrm{~cm}^{-1}$. The FTIR spectrum of silicone oil and different concentration of MXene nanoparticles shows an almost identical peak. A similar spectrum peak indicates that there is only physical interaction between the $\mathrm{Ti}_{3} \mathrm{C}_{2}$ nanoparticles and silicone oil [59]. Kotia, Haldar [59] reported similar findings in their research where the peak for the base fluid is identical with the different concentration of nanofluid. Hence, it can be concluded that the dispersion between $\mathrm{Ti}_{3} \mathrm{C}_{2}$ nanoparticles and silicone oil is chemically stable [59].

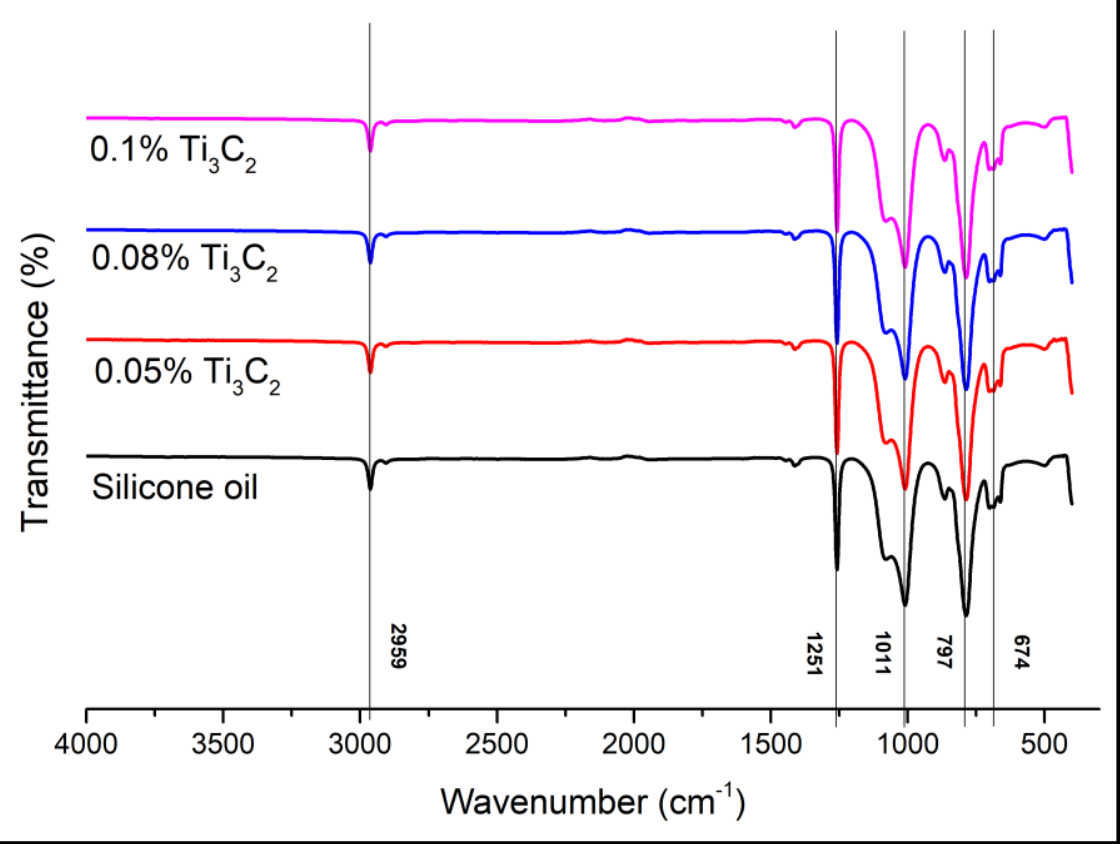

Figure 9. FTIR spectrum of Silicone oil, and Silicone oil-based MXene nanofluids with loading concentration of 0.05 wt. $\%, 0.08$ wt. $\%$ and 0.1 wt. $\%$ in the frequency range of $4000-450 \mathrm{~cm}^{-1}$

The major absorption peaks for the silicone oil and different concentration mixture of MXene nanoparticles occurred at $2959 \mathrm{~cm}^{-1}, 1251 \mathrm{~cm}^{-1}, 1011 \mathrm{~cm}^{-1}, 797 \mathrm{~cm}^{-1}$, and $674 \mathrm{~cm}^{-1}$. Table 2 shows

592 the wavenumbers of peak and the functional group at the respective wavenumbers. The peak 2959 $\mathrm{cm}^{-1}$ shows the $\mathrm{C}-\mathrm{H}$ bonding of the silicone oil based-fluid [60]. The peak defines that the stable 
594 balance of attractive and repulsive forces between carbon and hydrogen atoms exists in silicone 595 based-fluid [61].

596 The peaks $1251 \mathrm{~cm}^{-1}$ and $1011 \mathrm{~cm}^{-1}$ indicate the Si-O-Si bonding [60]. These peaks solely 597 attributed to silicone oil and do not overlap with the nanoparticles. The Si-O-Si bond had slightly 598 shifted to higher wavenumber due to the weak interaction of Silicone and Carbon molecules [62]. 599 The reason for the peak similarity on silicone oil and different concentration mixture of $\mathrm{Ti}_{3} \mathrm{C}_{2}$ is 600 because of the dominance of silicone oil's chemical structure [63].

601 The low-frequency peak of $797 \mathrm{~cm}^{-1}$ and $674 \mathrm{~cm}^{-1}$ in silicone oil and different concentration 602 mixture of $\mathrm{Ti}_{3} \mathrm{C}_{2}$ nanoparticles are caused by Si-C bonding [60]. In their research, Canaria, Lees 603 [64] mentioned that the Si-C bonding are expected to appear at lower frequencies due to the 604 stretching mode. From the observation of the entire peak, it can be concluded that there are no 605 peaks in the spectrum of nanoparticles corresponding to its component, silicone oil. From the FTIR 606 results, it has been proven that there is no chemical interaction that changes the nature of silicone 607 oil's functional group [65].

608 609 Table 2. Functional group at certain wavenumber

\begin{tabular}{|l|l|}
\hline $\begin{array}{l}\text { Wavenumbers, } \\
\left(\mathrm{cm}^{-1}\right)\end{array}$ & Functional groups \\
\hline 2959 & C-H bonding \\
\hline 1251 & Si-O-Si bonding \\
\hline 1011 & Si-O-Si bonding \\
\hline 797 & Si-C bonding \\
\hline 674 & Si-C bonding \\
\hline
\end{tabular}

610

\section{3.5. UV-Vis absorption of silicone oil-based MXene}

612 Figure 10 shows (RT) UV-Vis absorption spectra of silicone oil and different concentration of 613 MXene $\left(\mathrm{Ti}_{3} \mathrm{C}_{2}\right)$ nanoparticles dispersed into silicone oil at room temperature. The wavelength for 614 the silicone oil and the different concentration of nanofluids are near to $280 \mathrm{~nm}$ wavelength. 615 Azzolini, Docchio [66] found the similar value of the wavelength for the silicone oil in their 
research which is in the range of $280 \mathrm{~nm}$. In another research by Kawaguchi, Ohmura [67], the wavelength of silicone oil was found at $288 \mathrm{~nm}$. Authors stated that the wavelength of silicone oil found due to the emission of silicone oil which is adhered to the surface.

Figure 11 shows the absorption value of the percentage of MXene nanoparticle dispersed into silicone oil according to the weight percentage. It is observed in the spectra in Figure 11 that the overall absorption increased with the concentration of nanoparticle in silicone oil. The BeerLambert-Law states that when the concentration of a substance in a solution increases, the absorbance will increases too [68]. The peak value for the absorption for silicone oil, 0.05 wt.\% concentration of MXene, 0.08 wt.\% concentration of MXene, and 0.1 wt.\% concentration of MXene is $0.39,0.56,0.71$ and 0.92 , respectively. The percentage of absorption for the different concentrations is calculated using Eq (20).

Absorption percentage $(\%)=\frac{\text { Absorption of nanofluid-Absorption of base fluid }}{\text { Absorption of base fluid }} \times 100$

Eq. 20

The percentage of absorption of $0.05 \mathrm{wt} \%$ concentration of MXene is $43.6 \%$ higher than the base fluid, silicone oil. Meanwhile, the 0.08 wt. \% concentration of the $\mathrm{Ti}_{3} \mathrm{C}_{2}$ absorption percentage is $82.1 \%$ higher than silicone oil. As for $0.1 \mathrm{wt}$.\% concentration of $\mathrm{Ti}_{3} \mathrm{C}_{2}$ its absorption is $135.9 \%$ higher than silicone oil. The increment in absorption is directly proportional to the concentration of a substance in a base fluid which is proven by the Beer-Lambert-Law.

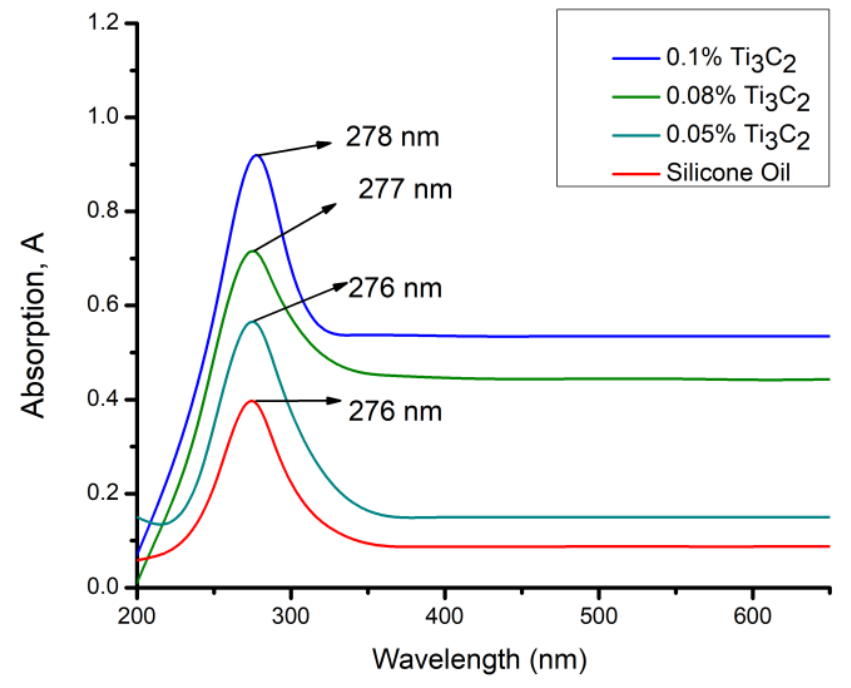


Figure 10. Variation of absorption with the wavelength of Silicone oil and different volume of $\mathrm{Ti}_{3} \mathrm{C}_{2}$ nanofluid

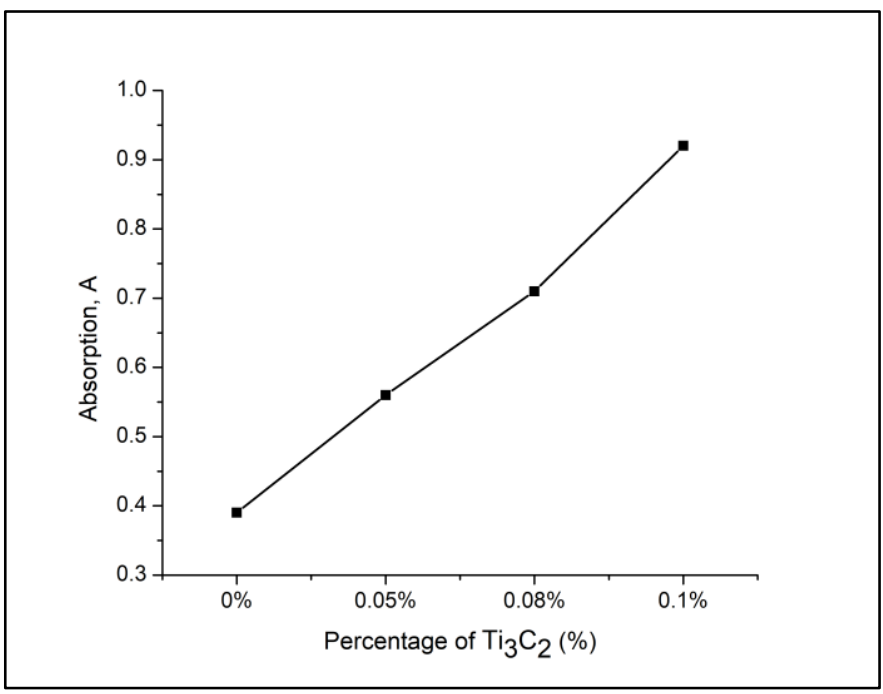

Figure 11. Absorption value of the percentage of $\mathrm{Ti}_{3} \mathrm{C}_{2}$ nanoparticle dispersed into silicone oil

\subsection{Morphological characteristics of MXene $\left(\mathrm{Ti}_{3} \mathrm{C}_{2}\right)$}

The FESEM images of the multilayered MXene are shown in Figure 12 (a, b). The insert further demonstrates that the thickness of the layered structure is very small. Flake-like structure of the multilayered MXene is clear from Figure $12(\mathrm{a}, \mathrm{b})$ which proves the completion of the exfoliation process. HRTEM images of the multilayered MXene are illustrated in Figure 12 (c, d). HRTEM images are in accordance with FESEM images indicating the multilayered structure of the MXene. Figure 12 (c) shows that the sheets of the MXene are very thin and transparent. Additionally, some wrinkles on the sheets are observed which might be due to the flexibility of MXene nanosheets [69]. Figure 12 (d) demonstrates a high-resolution TEM (HRTEM) image of a typical MXene nanosheet including the corresponding selected area electron diffraction (SAED) pattern. Its Fast Fourier Transform (FTT) (Figure $12 \mathrm{~d}$ ) reveals a hexagonal-based crystal with chain-like features of the MXene nanosheets [70]. The image clearly shows that the atomic arrangement in the basal planes is identical to that in the parent MAX phase [71]. HRTEM and FESEM images prove further convincing evidence for three-dimensional to the two-dimensional conversion of the material. From the acquired images from HRTEM, it is clear that the MXene sheets are more stable than 
graphene sheets under $200 \mathrm{kV}$ electron beam [72]. Energy dispersive X-Ray diffraction (EDX) was performed using HRTEM images to evaluate the intensity of the elements. Elemental analysis was conducted for 5 points. Atomic percentage for titanium is achieved in mean value of $75.90 \%$.

660 Mean atomic percentages of the other elements including aluminium, fluorine, oxygen and carbon 661 was $1.46,8.18,6.03$ and $8.43 \%$ respectively. The particle size of the as-synthesized MXene flakes 662 is in the range of $1-10 \mu \mathrm{m}$ as reported in our previous research work [35].
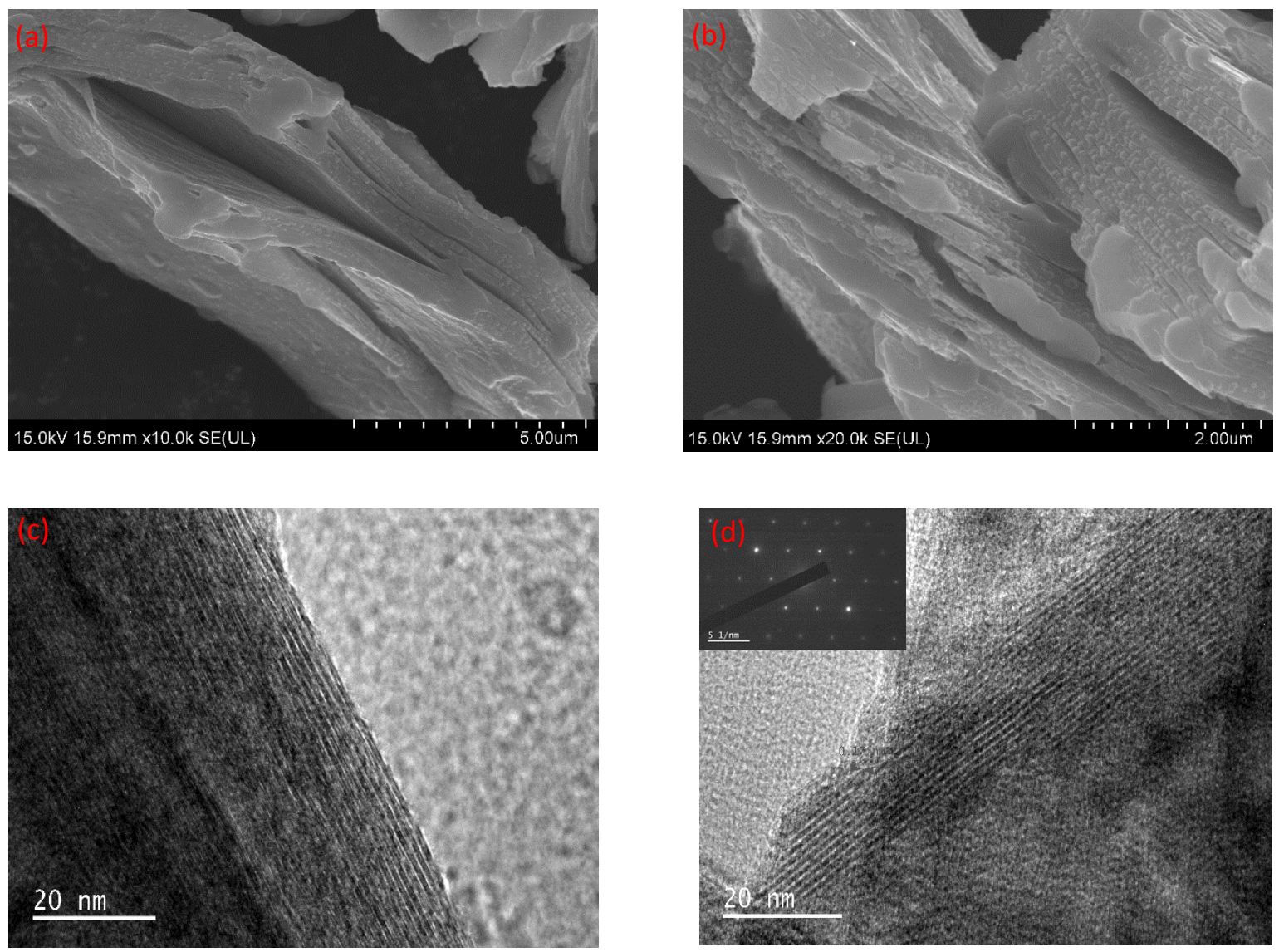

Figure 12. FESEM images of MXene flakes $(a, b)$ and HRTEM images of MXene flakes (c,d)

The experimental values of the thermal conductivity and viscosity will be introduced in the numerical solution. The dependence of the values of the thermal conductivity and the viscosity of 
670 the silicon oil/MXene nanofluid on the temperature at different nanoparticles loadings were

671 derived from the experimentally measured values as mentioned in Table 3 and Table 4.

672

673 Table 3. Derived correlations for the thermal conductivity of the silicone oil/MXene nanofluid at 674 different concentrations

\begin{tabular}{|c|c|}
\hline$\phi($ wt. $\%)$ & Derived correlation \\
\hline 0 & $k_{c f}=0.232 T_{c f}+131.4$ \\
\hline 0.05 & $k_{c f}=0.124 T_{c f}+178.3$ \\
\hline 0.08 & $k_{c f}=0.0000480 T_{c f}{ }^{3}-0.0112571 T_{c f}{ }^{2}+1.0485714 T_{c f}+165$ \\
\hline 0.1 & $k_{c f}=-0.0000026 T_{c f}{ }^{4}+0.000864 T_{c f}{ }^{3}-0.1056 T_{c f}{ }^{2}+5.86 T_{c f}+109$ \\
\hline
\end{tabular}

${ }^{*} \mathrm{k}$ in $(\mathrm{mW} / \mathrm{m} . \mathrm{K})$ and $\mathrm{T}$ in $\left({ }^{\circ} \mathrm{C}\right)$

676

677

678

679 680

Table 4. Derived correlations for the viscosity of the silicone oil/MXene nanofluid at different concentrations

\begin{tabular}{|c|c|}
\hline$\phi($ wt. \% $)$ & Derived correlation \\
\hline 0 & $\mu_{c f}=0.0011233 T_{c f}{ }^{4}-0.5348213 T_{c f}{ }^{3}+104.2939333 T_{c f}{ }^{2}$ \\
& $-10666.3966667 T_{c f}+547043$ \\
\hline 0.05 & $\mu_{c f}=0.0011279 T_{c f}{ }^{4}-0.5433173 T_{c f}{ }^{3}+106.1622667 T_{c f}{ }^{2}$ \\
& $-10800.3466666 T_{c f}+550215$ \\
\hline 0.08 & $\mu_{c f}=0.0015316 T_{c f}{ }^{4}-0.6624800 T_{c f}{ }^{3}+118.2451333 T_{c f}{ }^{2}$ \\
& $-11283.37 T_{c f}+556925$ \\
\hline 0.1 & $\mu_{c f}=0.0015884 T_{c f}{ }^{4}-0.6675680 T_{c f}{ }^{3}+117.0008667 T_{c f}{ }^{2}$ \\
&
\end{tabular}

$* \mu$ in $(\mu \mathrm{Pa} . \mathrm{s})$ and $T_{c f}$ in $\left({ }^{\circ} \mathrm{C}\right)$

682

Figure 13 shows the electrical performance of a hybrid CPVT system with the silicon oil/MXene nanofluid for cooling of the PV module. The figure shows the comparison of the 
electrical performance at different nanoparticles loadings and different solar concentrations. It is obvious from the figure that the electrical efficiency in general decreases with the increase of the solar concentration, which can be attributed to the great increase in the denominator (the input energy) in comparison to the nominator (the output electrical energy). On the other side, introducing more MXene nanoparticles enhances the electrical efficiency because of enhancing the cooling of the PV module as can be seen from Figure 13. The enhancement is found more pronounced at the higher solar concentrations. The electrical energy output showed different behavior. The electrical energy output is increased with the increase of the solar concentration due to the increase in the input energy. Moreover, it is increased with the nanoparticles loadings as a results of PV cooling enhancement. However, the electrical energy output from the PV module has started to decrease at the high concentration of eight due to insufficient cooling by the cooling fluid. This consequently degrades electrical performance of the system.

Figure 14 shows the thermal performance of the hybrid CPVT system at different nanoparticles loadings and different solar concentrations. Similar behavior like the electrical efficiency can be seen in the thermal efficiency. The thermal efficiency is decreased with the solar concentration due to higher rate of increase in the input energy with the solar concentration compared to output thermal energy. The thermal efficiency has enhanced by introducing more MXene nanoparticles to the silicon oil. The extracted thermal energy is increased with the increase in the solar concentration and the nanoparticles loading due to the heat up of the PV panel from one side and the enhancement of the thermal properties of the silicon oil with the addition of the MXene nanoparticles from the other side. With the higher values of solar concentrations, at which the effect of the nanoparticles loadings becomes more significant compared to its effect at the low solar concentrations.

Figure 15 shows the influence of the solar concentration and the nanoparticles loading on the average PV temperature and how it was reflected on the temperature gain through the cooling fluid. The increase in the solar concentration resulted in boosting the average temperature of the PV module, and its consequent improvement in the amount heat collected by the cooling fluid. On the other side, and due to enhancement of the thermal properties of the nanofluid, addition of more MXene nanoparticles resulted in better cooling for the PV module and again an improvement in the amount of heat collected by the cooling fluid. The effect of the solar concentration and the 
nanoparticles loading on the average PV temperature and the temperature gain through the cooling

716 fluid were reflected on the electrical and thermal performance of the system as discussed earlier.
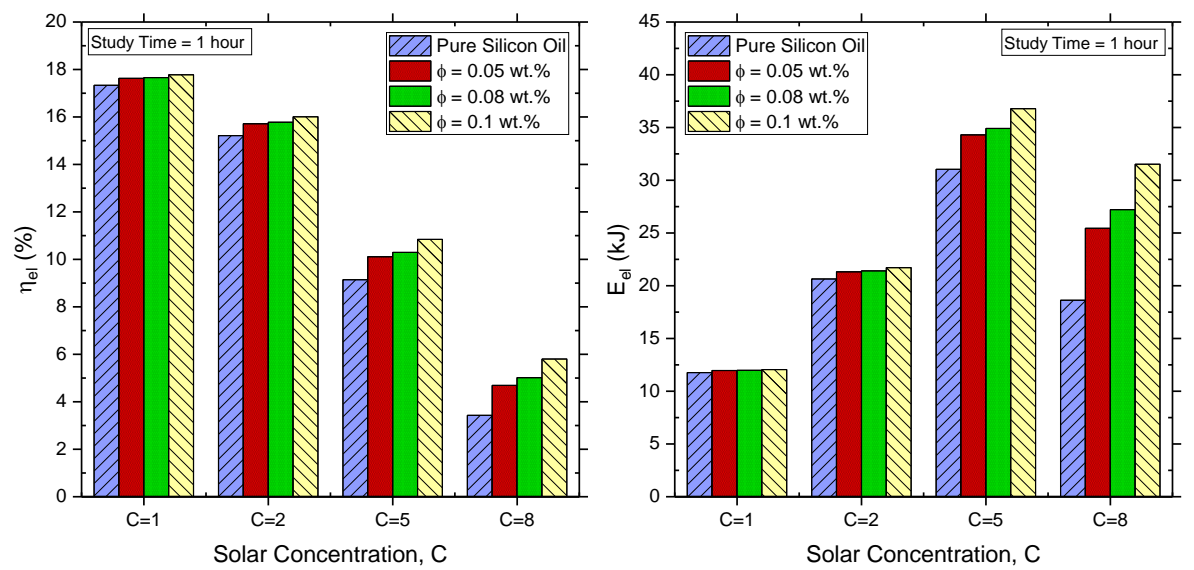

718 Figure 13. Electrical performance of the CPVT at different nanoparticles loadings and solar concentration
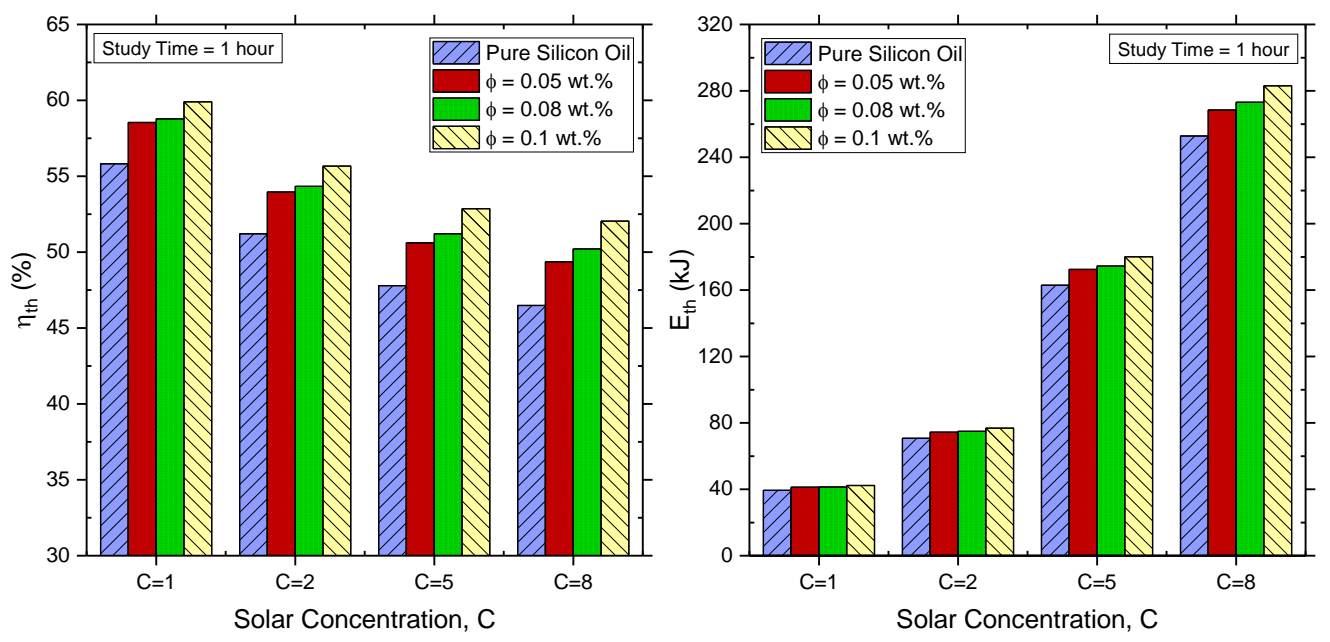

Figure 14. Thermal performance of the CPVT at different nanoparticles loadings and solar 

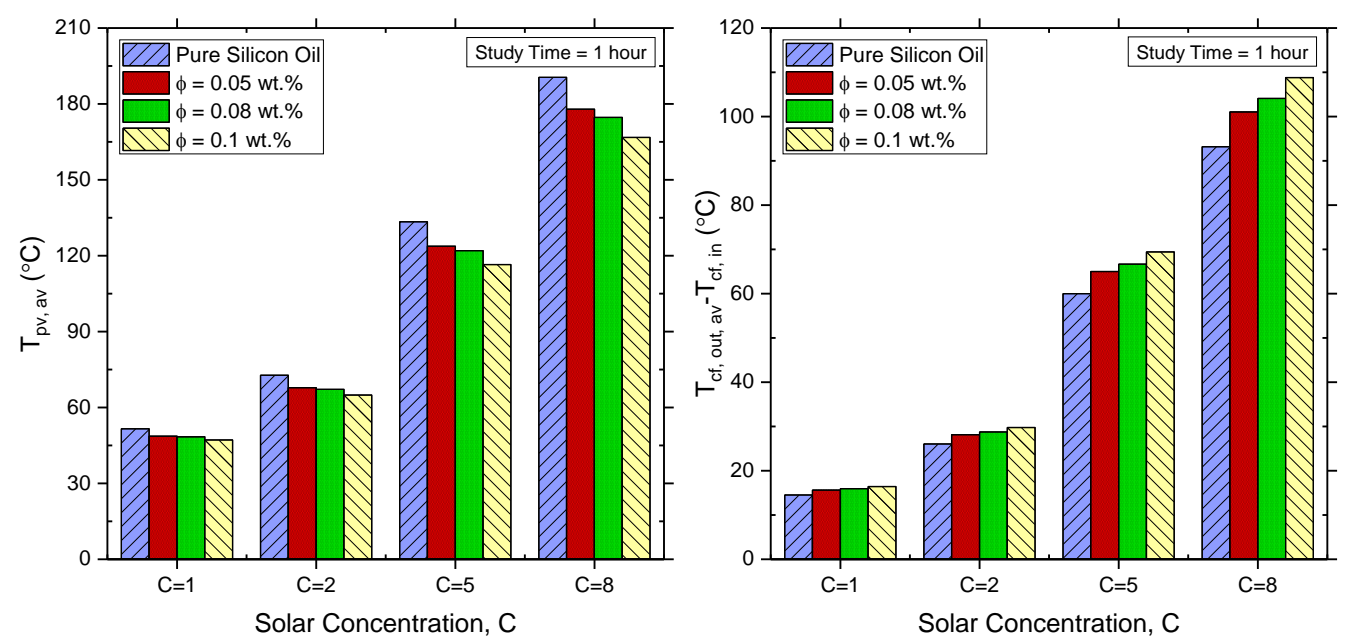

723 levels.

Figure 15. Average PV temperature of the CPVT and the temperature increase across the cooling fluid at different nanoparticles loadings and solar concentration

\section{Table 5 shows the effect of the MXene nanoparticles loading on percentage reduction in the} average temperature of the PV module at different solar concentrations. In addition, it clarifies the consequent enhancement percentage in the thermal and electrical energy outputs. The reduction and enhancement percentage are calculated for different nanoparticles loadings and solar concentrations. For the same solar concentration, more reduction percentage is achieved in the average PV module temperature at higher nanoparticles loadings, which resulted in better enhancement percentages in the overall electrical and thermal performance as a result of better cooling for the PV module. In contrast, fixing the value of the nanoparticles loading shows that the addition of the MXene nanoparticles becomes more efficient in enhancing the overall performance at higher solar concertation values. To sum up, MXene nanoparticle is a highly promising material that is more suitable to be used at high solar concentrations and temperature 
Table 5. Percentage of enhancement in the performance of the CPVT system at different nanoparticles loadings and solar concentration

\begin{tabular}{|c|c|c|c|c|c|}
\hline $\begin{array}{c}\text { Concentration } \\
\text { ratio }\end{array}$ & Change & Parameter & $\phi=0.05$ wt. $\%$ & $\phi=0.08$ wt. $\%$ & $\phi=0.1 \mathrm{wt} . \%$ \\
\hline \multirow{6}{*}{1} & $\%$ Reduction in & $\mathrm{T}_{\mathrm{pv}, \mathrm{av}}$ & 5.698 & 6.230 & 8.580 \\
\hline & \multirow{5}{*}{$\%$ Enhancement in } & $\eta_{\mathrm{el}}$ & 1.699 & 1.857 & 2.558 \\
\hline & & $\eta_{\text {th }}$ & 4.889 & 5.307 & 7.325 \\
\hline & & $\mathrm{E}_{\mathrm{el}}$ & 1.700 & 1.860 & 2.560 \\
\hline & & $\mathrm{Q}_{\text {th }}$ & 4.840 & 5.250 & 7.254 \\
\hline & & $\mathrm{T}_{\mathrm{cf}, \mathrm{out}, \mathrm{av}}-\mathrm{T}_{\mathrm{cf}, \text { in }}$ & 7.637 & 9.800 & 13.100 \\
\hline \multirow{6}{*}{2} & $\%$ Reduction in & $\mathrm{T}_{\mathrm{pv}, \mathrm{av}}$ & 6.779 & 7.640 & 10.830 \\
\hline & \multirow{5}{*}{$\%$ Enhancement in } & $\eta_{\mathrm{el}}$ & 3.245 & 3.660 & 5.186 \\
\hline & & $\eta_{\text {th }}$ & 5.385 & 6.103 & 8.722 \\
\hline & & $\mathrm{E}_{\mathrm{el}}$ & 3.240 & 3.660 & 5.190 \\
\hline & & $\mathrm{Q}_{\mathrm{th}}$ & 5.330 & 6.030 & 8.619 \\
\hline & & $\mathrm{T}_{\mathrm{cf}, \text { out, av }}-\mathrm{T}_{\mathrm{cf}, \mathrm{in}}$ & 8.015 & 10.420 & 14.270 \\
\hline \multirow{6}{*}{5} & $\%$ Reduction in & $\mathrm{T}_{\mathrm{pv}, \mathrm{av}}$ & 7.212 & 8.570 & 12.690 \\
\hline & \multirow{5}{*}{$\%$ Enhancement in } & $\eta_{\mathrm{el}}$ & 10.530 & 12.510 & 18.530 \\
\hline & & $\eta_{\text {th }}$ & 5.892 & 7.136 & 10.61 \\
\hline & & $\mathrm{E}_{\mathrm{el}}$ & 10.53 & 12.510 & 18.530 \\
\hline & & $\mathrm{Q}_{\mathrm{th}}$ & 5.860 & 7.090 & 10.550 \\
\hline & & $\mathrm{T}_{\mathrm{cf}, \mathrm{out}, \mathrm{av}}-\mathrm{T}_{\mathrm{cf}, \mathrm{in}}$ & 8.290 & 11.070 & 15.690 \\
\hline \multirow{6}{*}{8} & $\%$ Reduction in & $\mathrm{T}_{\mathrm{pv}, \mathrm{av}}$ & 6.593 & 8.300 & 12.450 \\
\hline & \multirow{5}{*}{$\%$ Enhancement in } & $\eta_{\mathrm{el}}$ & 36.670 & 46.180 & 69.240 \\
\hline & & $\eta_{\text {th }}$ & 6.184 & 8.017 & 11.950 \\
\hline & & $\mathrm{E}_{\mathrm{el}}$ & 36.670 & 46.180 & 69.240 \\
\hline & & $\mathrm{Q}_{\mathrm{th}}$ & 6.170 & 7.990 & 11.920 \\
\hline & & $\mathrm{T}_{\mathrm{cf}, \text { out }, \mathrm{av}}-\mathrm{T}_{\mathrm{cf}, \mathrm{in}}$ & 8.445 & 11.730 & 16.780 \\
\hline
\end{tabular}

MXene based silicone oil is formulated for the first time to evaluate thermal properties applicable to a CPVT system. About $64 \%$ of thermal conductivity improvement is found for the 0.1 wt.\% concentration of MXene in silicone oil at $150^{\circ} \mathrm{C}$ compared to pure silicone oil. This is a 
remarkable achievement in terms of thermal conductivity improvement. The Authors found that viscosity does not change with the addition of MXene into silicone oil. Moreover, viscosity found to be reduced with an increase in temperature. On average, viscosity is found to be reduced by $32 \%$ for $25^{\circ} \mathrm{C}$ increase in temperature. This is also outstanding finding as viscosity reduction is a desirable requirement since this will reduce pumping power in a flow channel. Silicone oil-based MXene nanofluid with 0.1 wt.\% concentration is thermally stable up to $\sim 380{ }^{\circ} \mathrm{C}$. The highest electrical efficiency of CPVT is obtained for the $0.1 \mathrm{wt} . \%$ concentration of MXene into silicone oil for all concentration ratio. However, electrical efficiencies are low at a higher concentration ratio. More thermal heat can be gained for a higher concentration ratio. This heat can be useful for various process heat applications.

The major absorption peaks for the silicone oil and different concentration of MXene nanoparticles have been obtained at $2959 \mathrm{~cm}^{-1}, 1251 \mathrm{~cm}^{-1}, 1011 \mathrm{~cm}^{-1}, 797 \mathrm{~cm}^{-1}$, and $674 \mathrm{~cm}^{-1}$. The percentage of the absorption for 0.05 wt.\%, 0.08wt.\% and 0.1wt.\% MXene are, 43.6\%, 82.1, and $135.9 \%$, respectively than pure silicone oil. The mean atomic percentage for titanium is achieved to be $75.90 \%$ with carbon content $8.43 \%$. Future works can be considered for this novel nanofluid including density measurement, calculation of pumping factor in real concentrated photovoltaic systems and thermal conductivity and viscosity measurement at high temperature.

Acknowledgement: "R. Saidur would like to acknowledge the financial support provided by the Sunway University through the project no\# STR-RCTR-RCNMET-001-2019". The authors would like to show their gratitude to Dr. Michael Naguib for providing MXene and some other advice.

\section{References}

1. Choi, S., D. Singer, and H. Wang, Developments and applications of non-Newtonian flows. ASME FED, 1995. 66: p. 99-105.

2. Mehrali, M., M.K. Ghatkesar, and R. Pecnik, Full-spectrum volumetric solar thermal conversion via graphene/silver hybrid plasmonic nanofluids. Applied energy, 2018. 224: p. 103-115.

3. Aslfattahi, N., et al., Experimental Investigation of Thermal Stability and Enthalpy of Eutectic Alkali Metal Solar Salt Dispersed with MgO Nanoparticles. 2019. 10(6): p. 1112-1119.

4. Murshed, S., K. Leong, and C. Yang, Investigations of thermal conductivity and viscosity of nanofluids. International Journal of Thermal Sciences, 2008. 47(5): p. 560-568.

5. Wang, F., et al., Surfactant-free ionic liquid-based nanofluids with remarkable thermal conductivity enhancement at very low loading of graphene. Nanoscale research letters, 2012. 7(1): p. 314. 
6. Jiang, Z., et al., Novel key parameter for eutectic nitrates based nanofluids selection for concentrating solar power (CSP) system. Applied energy, 2019. 235: p. 529-542.

7. Bakthavatchalam, B., et al., Comprehensive study on nanofluid and ionanofluid for heat transfer enhancement: A review on current and future perspective. 2020: p. 112787.

8. Liu, M.-S., et al., Enhancement of thermal conductivity with $\mathrm{Cu}$ for nanofluids using chemical reduction method. International Journal of Heat and Mass Transfer, 2006. 49(17-18): p. 30283033.

9. Karthikeyan, N., J. Philip, and B. Raj, Effect of clustering on the thermal conductivity of nanofluids. Materials Chemistry and Physics, 2008. 109(1): p. 50-55.

10. Kim, S.H., S.R. Choi, and D. Kim, Thermal conductivity of metal-oxide nanofluids: particle size dependence and effect of laser irradiation. Journal of Heat Transfer, 2007. 129(3): p. 298-307.

11. Daneshazarian, R., et al., Concentrating photovoltaic thermal (CPVT) collectors and systems: Theory, performance assessment and applications. Renewable and Sustainable Energy Reviews, 2018. 81: p. 473-492.

12. Krishna, Y., et al., State-of-the-art heat transfer fluids for parabolic trough collector. 2020. 152: p. 119541.

13. $\mathrm{Xu}, \mathrm{Z}$. and $\mathrm{C}$. Kleinstreuer, Concentration photovoltaic-thermal energy co-generation system using nanofluids for cooling and heating. Energy Conversion and Management, 2014. 87: p. 504512.

14. Kleinstreuer, C. and Y. Feng, Experimental and theoretical studies of nanofluid thermal conductivity enhancement: a review. Nanoscale research letters, 2011. 6(1): p. 229.

15. Mahian, O., et al., A review of the applications of nanofluids in solar energy. International Journal of Heat and Mass Transfer, 2013. 57(2): p. 582-594.

16. Subramani, J., et al., Efficiency and heat transfer improvements in a parabolic trough solar collector using TiO2 nanofluids under turbulent flow regime. Renewable energy, 2018. 119: p. 19-31.

17. Tyagi, H., P. Phelan, and R. Prasher, Predicted efficiency of a low-temperature nanofluid-based direct absorption solar collector. Journal of solar energy engineering, 2009. 131(4).

18. Saidur, R., et al., Evaluation of the effect of nanofluid-based absorbers on direct solar collector. International Journal of Heat and Mass Transfer, 2012. 55(21-22): p. 5899-5907.

19. Taylor, R.A., et al., Applicability of nanofluids in high flux solar collectors. Journal of Renewable and Sustainable Energy, 2011. 3(2): p. 023104.

20. Otanicar, T.P., et al., Nanofluid-based direct absorption solar collector. Journal of renewable and sustainable energy, 2010. 2(3): p. 033102.

21. Han, X., et al., Performance improvement of a PV/T system utilizing Ag/CoSO4-propylene glycol nanofluid optical filter. 2020. 192: p. 116611.

22. Abdelrazik, A., et al., Optical behavior of a water/silver nanofluid and their influence on the performance of a photovoltaic-thermal collector. 2019. 201: p. 110054.

23. Abdelrazik, A.S., et al., Evaluation of the effects of optical filtration and nanoPCM on the performance of a hybrid photovoltaic-thermal solar collector. 2019. 195: p. 139-156.

24. Crisostomo, F., et al., A hybrid PV/T collector using spectrally selective absorbing nanofluids. 2017. 193: p. 1-14.

25. An, W., et al., Experimental investigation of a concentrating PV/T collector with Cu9S5 nanofluid spectral splitting filter. 2016. 184: p. 197-206.

26. Naguib, M., et al., Two-dimensional nanocrystals produced by exfoliation of Ti3AIC2. Advanced Materials, 2011. 23(37): p. 4248-4253.

27. $\mathrm{Li}, \mathrm{M}$. and B. Mu, Effect of different dimensional carbon materials on the properties and application of phase change materials: A review. Applied energy, 2019. 242: p. 695-715. 
28. Naguib, M., et al., Two-dimensional transition metal carbides. ACS nano, 2012. 6(2): p. 13221331.

29. Naguib, M., et al., New two-dimensional niobium and vanadium carbides as promising materials for Li-ion batteries. Journal of the American Chemical Society, 2013. 135(43): p. 15966-15969.

30. Ghidiu, M., et al., Synthesis and characterization of two-dimensional Nb 4 C 3 (MXene). Chemical Communications, 2014. 50(67): p. 9517-9520.

31. Pang, J., et al., Applications of 2D MXenes in energy conversion and storage systems. Chemical Society reviews, 2019. 48(1): p. 72-133.

32. Sun, D., et al., Two-dimensional Ti3C2 as anode material for Li-ion batteries. Electrochemistry Communications, 2014. 47: p. 80-83.

33. Hu, Q., et al., MXene: a new family of promising hydrogen storage medium. The Journal of Physical Chemistry A, 2013. 117(51): p. 14253-14260.

34. Khazaei, M., et al., Novel electronic and magnetic properties of two-dimensional transition metal carbides and nitrides. Advanced Functional Materials, 2013. 23(17): p. 2185-2192.

35. Aslfattahi, N., et al., Experimental investigation of energy storage properties and thermal conductivity of a novel organic phase change material/MXene as A new class of nanocomposites. 2020. 27: p. 101115.

36. Moretto, H.H., M. Schulze, and G. Wagner, Silicones. Ullmann's encyclopedia of industrial chemistry, 2000.

37. Parel, J.-M.A., et al., Silicone oils: physicochemical properties, in Retina: Fourth Edition. 2006, Elsevier Inc. p. 2191-2210.

38. Chen, L. and H. Xie, Silicon oil based multiwalled carbon nanotubes nanofluid with optimized thermal conductivity enhancement. Colloids and Surfaces A: Physicochemical and Engineering Aspects, 2009. 352(1-3): p. 136-140.

39. Maxwell, J.C., A treatise on electricity and magnetism. Vol. 1. 1873: Oxford: Clarendon Press.

40. Cengel, Y. and A. Ghajar, Chapter eight: internal forced convection, in Heat and Mass Transfer, Fundamentals and Applications. 2011, McGraw-Hill New York. p. 489.

41. Baby, T.T. and S. Ramaprabhu, Investigation of thermal and electrical conductivity of graphene based nanofluids. Journal of Applied Physics, 2010. 108(12): p. 124308.

42. Mehrali, M., et al., Investigation of thermal conductivity and rheological properties of nanofluids containing graphene nanoplatelets. Nanoscale research letters, 2014. 9(1): p. 15.

43. Hammami, A., N. Raymond, and M. Armand, Lithium-ion batteries: Runaway risk of forming toxic compounds. Nature, 2003. 424(6949): p. 635.

44. Sarafraz, M. and M. Safaei, Diurnal thermal evaluation of an evacuated tube solar collector (ETSC) charged with graphene nanoplatelets-methanol nano-suspension. Renewable Energy, 2019. 142: p. 364-372.

45. Anasori, B., M.R. Lukatskaya, and Y. Gogotsi, 2D metal carbides and nitrides (MXenes) for energy storage. Nature Reviews Materials, 2017. 2(2): p. 16098.

46. Gu, J., et al., Thermal percolation behavior of graphene nanoplatelets/polyphenylene sulfide thermal conductivity composites. Polymer Composites, 2014. 35(6): p. 1087-1092.

47. Xuan, Y., Q. Li, and W. Hu, Aggregation structure and thermal conductivity of nanofluids. AIChE Journal, 2003. 49(4): p. 1038-1043.

48. Sen Gupta, S., et al., Thermal conductivity enhancement of nanofluids containing graphene nanosheets. Journal of Applied Physics, 2011. 110(8): p. 084302.

49. Shahil, K.M. and A.A. Balandin, Thermal properties of graphene and multilayer graphene: Applications in thermal interface materials. Solid State Communications, 2012. 152(15): p. 13311340. 
50. Aguilar, T., et al., Investigation of enhanced thermal properties in NiO-based nanofluids for concentrating solar power applications: A molecular dynamics and experimental analysis. Applied energy, 2018. 211: p. 677-688.

51. Sarafraz, M., et al., Experimental Investigation on Thermal Performance of a PV/T-PCM (Photovoltaic/Thermal) System Cooling with a PCM and Nanofluid. Energies, 2019. 12(13): p. 2572.

52. Rashin, M.N. and J. Hemalatha, Synthesis and viscosity studies of novel ecofriendly ZnO-coconut oil nanofluid. Experimental Thermal and Fluid Science, 2013. 51: p. 312-318.

53. Cardellini, A., et al., Thermal transport phenomena in nanoparticle suspensions. Journal of Physics: Condensed Matter, 2016. 28(48): p. 483003.

54. Mahian, O., et al., Nanofluids effects on the evaporation rate in a solar still equipped with a heat exchanger. Nano Energy, 2017. 36: p. 134-155.

55. Patterson, R., et al., Handbook of Thermoset Plastics: 17. Crosslinked Thermoplastics. 2013: Elsevier Inc. Chapters.

56. Yao, F., et al., Thermal decomposition kinetics of natural fibers: activation energy with dynamic thermogravimetric analysis. 2008. 93(1): p. 90-98.

57. Brydson, J.A., Plastics materials. 1999: Elsevier.

58. Zhou, W.-y., et al., Heat Conductive Composites Silicone Rubber. Polymer Materials Science and Engineering, 2007. 23(4): p. 242.

59. Kotia, A., et al., Effect of copper oxide nanoparticles on thermophysical properties of hydraulic oil-based nanolubricants. Journal of the Brazilian Society of Mechanical Sciences and Engineering, 2017. 39(1): p. 259-266.

60. Johnson, L.M., et al., Elastomeric microparticles for acoustic mediated bioseparations. Journal of nanobiotechnology, 2013. 11(1): p. 22.

61. Allahyarzadeh, V., et al., In situ synthesis of nano silver on polyester using NaOH/Nano TiO2. Journal of Applied Polymer Science, 2013. 129(2): p. 892-900.

62. Jayasekara, R., et al., Preparation, surface modification and characterisation of solution cast starch PVA blended films. Polymer testing, 2004. 23(1): p. 17-27.

63. Kim, C.H., et al., Instrumental studies on silicone oil adsorption to the surface of intraocular lenses. Applied Surface Science, 2012. 262: p. 146-152.

64. Canaria, C.A., et al., Characterization of the carbon-silicon stretch in methylated porous siliconobservation of an anomalous isotope shift in the FTIR spectrum. Inorganic Chemistry Communications, 2002. 5(8): p. 560-564.

65. Mansur, H.S., R.L. Oréfice, and A.A. Mansur, Characterization of poly (vinyl alcohol)/poly (ethylene glycol) hydrogels and PVA-derived hybrids by small-angle X-ray scattering and FTIR spectroscopy. Polymer, 2004. 45(21): p. 7193-7202.

66. Azzolini, C., et al., Interactions between light and vitreous fluid substitutes. Archives of Ophthalmology, 1992. 110(10): p. 1468-1471.

67. Kawaguchi, Y., H. Ohmura, and T. Sato, Detection of trace substances adhered to a metal surface by laser-induced breakdown spectroscopy. Journal of Analytical Atomic Spectrometry, 2017. 32(3): p. 609-615.

68. Kocsis, L., P. Herman, and A. Eke, The modified Beer-Lambert law revisited. Physics in Medicine \& Biology, 2006. 51(5): p. N91.

69. Cao, Y., et al., Enhanced thermal properties of poly (vinylidene fluoride) composites with ultrathin nanosheets of MXene. RSC Advances, 2017. 7(33): p. 20494-20501.

70. Zhou, J., et al., A two-dimensional zirconium carbide by selective etching of Al3C3 from nanolaminated Zr3AI3C5. Angewandte Chemie International Edition, 2016. 55(16): p. 50085013. 
927 71. Naguib, M., et al., 25th anniversary article: MXenes: a new family of two-dimensional materials. $928 \quad$ Advanced Materials, 2014. 26(7): p. 992-1005.

929 72. Storm, A., et al., Fabrication of solid-state nanopores with single-nanometre precision. Nature $930 \quad$ materials, 2003. 2(8): p. 537.

931 